\title{
Matching in Networks with Bilateral Contracts
}

\author{
By John William Hatfield and Scott Duke Kominers*
}

We introduce a model in which firms trade goods via bilateral contracts which specify a buyer, a seller, and the terms of the exchange. This setting subsumes (many-to-many) matching with contracts, as well as supply chain matching. When firms' relationships do not exhibit a supply chain structure, stable allocations need not exist. By contrast, in the presence of supply chain structure, a natural substitutability condition characterizes the maximal domain of firm preferences for which stable allocations always exist. Furthermore, the classical lattice structure, rural hospitals theorem, and one-sided strategy-proofness results all generalize to this setting.

The theoretical literature on two-sided matching began with the simple one-to-one (marriage) model of Gale and Shapley (1962), in which agents on opposite sides of a market (men and women) seek to match into pairs. The central solution concept in this literature is stability, the requirement that, if two agents are not matched to each other, at least one of them prefers his or her assigned partner to the other agent. Gale and Shapley (1962) showed that stable one-to-one matches exist in general, and obtained conditions under which this existence result is preserved even if agents on one side of the market are allowed to match to multiple partners, that is, when the matching is many-to-one (as in college admissions and doctor-hospital matching). Following high-profile applications of matching in labor markets and school choice programs, ${ }^{1}$ the foundational work on matching has been extensively generalized. ${ }^{2}$ Recently, Ostrovsky (2008) illustrated that

\footnotetext{
* Hatfield: Graduate School of Business, Stanford University, 655 Knight Way, Stanford, CA 94305 (email: hatfield@stanford.edu); Kominers: Harvard Business School, Harvard University, Wyss Hall, Soldiers Field, Boston MA 02163 (email: skominers@ @bs.edu). The authors are grateful for the helpful comments of Drew Fudenberg, Jason D. Hartline, Sonia Jaffe, Fuhito Kojima, Michael Ostrovsky, Alvin E. Roth, Alexander Westkamp, E. Glen Weyl, and seminar audiences at Chicago, Harvard, Northwestern, Stanford, the Duke ERID Conference on Roth and Sotomayor, and the 11th ACM Conference on Electronic Commerce. Hatfield appreciates the hospitality of Harvard Business School, which hosted him during parts of this research. Additionally, Kominers gratefully acknowledges the support of a National Science Foundation Graduate Research Fellowship, a Yahoo! Key Scientific Challenges Program Fellowship, a Terence M. Considine Fellowship in Law and Economics funded by the John M. Olin Center at Harvard Law School, and the Danielan Fund.

${ }^{1}$ Roth and Sotomayor (1990) provide a survey of the pre-1990 theory of matching. Roth (2008) gives an updated account, as well as references for historical and recent applications of matching. For examples of specific applications, see the work of Roth and Peranson (1999) (National Resident Matching Program) and Abdulkadiroğlu et al. (2005); Abdulkadiroğlu, Pathak and Roth $(2005,2009)$ (school choice)

${ }^{2}$ Kelso and Crawford (1982) extended many-to-one matching to a setting in which matches are supplemented by wage negotiations; Hatfield and Milgrom (2005) generalized this framework still further, by allowing agents to negotiate contracts which fully specify both a matching and the conditions of the match; the possibility of such a generalization was first noted by remarks of Crawford and Knoer (1981) and Kelso and Crawford (1982).

Meanwhile, a host of work has studied the existence of stable matchings in many-to-many matching settings, two-sided markets in which all agents may match to multiple partners (as in the matching of consultants to firms). Many-to-many matching has been studied, for example, in the work of Sotomayor (1999, 2004), Echenique and Oviedo (2006), and Konishi and Ünver (2006). Recently, Klaus and Walzl (2009) and Hatfield and Kominers (2010) merged this line of research with that of Hatfield and Milgrom (2005), introducing a theory of many-to-many matching with contracts.
} 
matching markets need not be two-sided-they may instead consist of a market of firms organized into supply chains. Earlier matching models easily embed into the Ostrovsky (2008) supply chain framework: for example, the many-to-one matching market between doctors and hospitals may be thought of as a "one-step supply-chain" in which doctors sell their services to hospitals.

Although the expanding work on matching has eliminated nearly all the theoretical restrictions imposed in the early literature, two assumptions have been maintained throughout, either implicitly or explicitly:

- acyclicity - no agent may both buy from and sell to another agent, even through intermediaries, and

- full substitutability - upon being endowed with an additional item, an agent's demand for other items is lower, both in the sense of a reduced desire to buy additional items and an increased desire to sell items he currently owns. ${ }^{3}$

The acyclicity condition is implicit in all studies of two-sided matching, as the "two sides" may be identifed as the set of buyers and sellers, and so each agent acts only as a buyer or only as a seller. Furthermore, acyclicity corresponds to the supply chain structure imposed by Ostrovsky (2008).

Full substitutability generalizes a heavily-studied notion of preference (gross) substitutability first introduced by Kelso and Crawford (1982). Substitutability, in turn, generalizes the responsive preference condition introduced by Roth (1985). These successively more-general substitutability conditions have been shown to be essential for the existence of stable allocations in a variety of matching contexts. ${ }^{4}$

Moreover, both acyclicity and full substitutability are natural in most previously-studied matching settings, such as the matching of residents to hospitals (Roth and Peranson (1999)), the assignment of students to schools (Abdulkadiroğlu et al. (2005); Abdulkadiroğlu, Pathak and Roth $(2005,2009))$, and the supply-chain production of steel (Ostrovsky (2008)). Acyclicity may not hold, however, in electricity markets, where an individual firm may buy power from a neighboring firm in one region and sell power to that same firm in another region. Full substitutability is unlikely to apply in settings such as the matching of auto-parts suppliers and assemblers, where different parts are complementary in the production of the final good. ${ }^{5}$

\footnotetext{
${ }^{3}$ Full substitutability is a condition on firms' preferences familiar from auction theory. Indeed, full substitutability is an ordinal analogue of the conventional notion of substitutability from auction theory (see, for instance, Milgrom (2004)) and we prove more formally that it is equivalent to quasisubmodularity of the associated indirect utility function.

${ }^{4}$ The sufficiency and necessity of substititutability for the guaranteed existence of stable allocations holds in the settings of many-to-one matching (Roth (1984) proves sufficiency; Hatfield and Kojima (2008) prove necessity), manyto-many matching (Roth (1984) and Echenique and Oviedo (2006) prove sufficiency; necessity follows from the results of Hatfield and Kojima (2008)), many-to-many matching with contracts (Klaus and Walzl (2009) and Hatfield and Kominers (2010) prove sufficiency; Hatfield and Kominers (2010) prove necessity). Substitutable preferences are sufficient for the existence of a stable outcome in the setting of many-to-one matching with contracts (Hatfield and Milgrom (2005)) but are not necessary (Hatfield and Kojima $(2008,2010)$ ).

${ }^{5}$ In an econometric study of many-to-many matching with transferable utility, Fox (2010) identifies substantial complementarity between inputs into automobile production.
} 
In this paper, we introduce a matching model in which firms trade via bilateral contracts which specify a buyer, a seller, and the terms of the exchange. This model subsumes all classical matching models, and its generality allows us to make two novel theoretical contributions.

First, we show that both acyclicity and full substitutability are necessary for classical matching theory. If either condition is violated, then stable allocations cannot be guaranteed. ${ }^{6}$ Intuitively, if a contracting relationship contains a cycle, and if a firm in the cycle has an outside option which the firm prefers to one contract in the cycle, then both the outside option and the complete trading cycle are unstable; the necessity of acyclicity follows. The necessity of full substitutability is more technical to illustrate, but follows closely upon prior results of Hatfield and Kominers (2010).

Second, in the presence of acyclicity and fully substitutable preferences, we fully generalize the key results of classical matching theory. We prove that, in the presence of acyclicity and full substitutability, stable allocations correspond bijectively to fixed points of an isotone operator; Tarski's fixed point theorem then guarantees the existence of a lattice of stable allocations. We also prove a generalization of the classical rural hospitals theorem of Roth (1986) and the strategy-proofness results of Hatfield and Milgrom (2005) and Hatfield and Kojima (2009). These latter results display a surprising structure which can only be elicited within a framework as general as ours: in particular, we show that for each agent the difference between the numbers of buy- and sell-contracts held by that agent, rather than the absolute number of contracts held, is invariant across stable allocations. ${ }^{7}$

In light of our necessity results, our work establishes a frontier of matching theory. Without acyclicity and fully substitutable preferences, stable allocations are not guaranteed to exist in general, and hence the results of classical matching theory fail. Up to the failure of these conditions, however, all of the results of classical matching theory hold. Thus, our work in some sense characterizes the set of applications to which classical matching theory applies without imposition of additional structure: Settings where both acyclicity and full substitutability hold—such as labor markets, school choice, and steel production—are directly approachable via classical matching theory; settings where either acyclicity or full substitutability fails—such as electricity and auto-parts marketsare not.

The remainder of this paper is organized as follows. We formalize our model, restrictions on preferences, and solution concepts in Section I. In Section II, we prove the sufficiency and necessity of fully substitutable preferences for the existence of stable con-

\footnotetext{
${ }^{6}$ Note that we use a notion of stability which is distinct from the concept of chain stability introduced by Ostrovsky (2008). Our stability concept is more stringent than chain stability, although these two notions coincide on acyclic contract domains over which firm preferences are fully substitutable (Theorem 7). As we detail in Section I.B, for domains where these conditions do not hold, chain stability has some unappealing properties.

${ }^{7}$ The Roth (1986) rural hospitals theorem and its subsequent generalizations by Hatfield and Milgrom (2005) and Hatfield and Kominers (2010) all show that, under certain conditions, the number of contracts signed by each agent is invariant across stable allocations. The natural conjecture that this exact result would extend to our setting is false, as we demonstrate in Section III.A. We instead find that the proper invariant for each firm in our framework is the difference between the numbers of buy- and sell-contracts held by that firm. This result implies the previous rural hospitals results because, in a two-sided market, no firm can be both a buyer and a seller.
} 
tract allocations. In Section III, we discuss the structure of the set of stable allocations, proving our rural hospitals and strategy-proofness results. We conclude in Section IV. All proofs are deferred to the Appendix.

\section{Model}

There is finite set $F$ of of firms, and a finite set $X$ of contracts. Each contract $x \in X$ is associated with both a buyer $x_{B} \in F$ and a seller $x_{S} \in F$; there may be several contracts with the same buyer and the same seller. ${ }^{8}$

For concreteness, one may suppose each contract $x \in X$ denotes the exchange of a single unit of a good from $x_{S}$ to $x_{B} \cdot{ }^{9}$ However, contracts need not use a constant unit. For example, labor markets might allow both full- and part-time job contracts. ${ }^{10}$

Let $x_{F} \equiv\left\{x_{B}, x_{S}\right\}$ be the set of the firms associated with contract $x$. For a set of contracts $Y$, we denote

$$
Y_{B} \equiv \bigcup_{y \in Y}\left\{y_{B}\right\}, \quad Y_{S} \equiv \bigcup_{y \in Y}\left\{y_{S}\right\}, \quad Y_{F} \equiv Y_{B} \cup Y_{S}
$$

The contract set $X$ is acyclic if there does not exist a cycle, i.e. a set of contracts

$$
\left\{x^{1}, \ldots, x^{N}\right\} \subseteq X
$$

such that $x_{B}^{1}=x_{S}^{2}, x_{B}^{2}=x_{S}^{3}, \ldots, x_{B}^{N-1}=x_{S}^{N}, x_{B}^{N}=x_{S}^{1}$ (as pictured in Figure 1). ${ }^{11}$ This condition is equivalent to the condition that there is an ordering $\triangleright$ on $F$ such that for all $x \in X, x_{S} \triangleleft x_{B}$; for an acyclic contract set $X$, if $f \triangleleft f^{\prime}$, we will say that $f$ is upstream of $f^{\prime}$ and that $f^{\prime}$ is downstream of $f$.

We say that $X$ is exhaustive if there is a contract between any two firms, that is, if for all $f \neq f^{\prime}, f, f^{\prime} \in F$ there exists a contract $x$ such that $x_{F}=\left\{f, f^{\prime}\right\}$.

Each $f \in F$ has a strict preference relation $P^{f}$ over sets of contracts involving $f$. Let $\left.Y\right|_{f} \equiv\left\{y \in Y: f \in y_{F}\right\}$ be the set of contracts in $Y$ associated with firm $f$.

For any $Y \subseteq X$, we first define the choice set of $f$ as the set of contracts $f$ chooses from $Y: 12$

$$
C^{f}(Y) \equiv \max _{P^{f}}\left\{Z \subseteq Y: x \in Z \Rightarrow f \in x_{F}\right\}
$$

\footnotetext{
${ }^{8}$ Note that since $X$ is finite, we may interpret $X$ as a subset of the set $F \times F \times T$, for some finite set $T$ of possible contract terms. With this notation, a contract $x \in X$ is a 3-tuple: $x=\left(x_{B}, x_{S}, t\right)$ with $x_{B}, x_{S} \in F$ and $t \in T$.

${ }^{9}$ In this case, an exchange of 17 units from $x_{S}$ to $x_{B}$ would technically occur through 17 different contracts. While in practice the actual sale would not transact in this fashion-a single contractual document would cover the sale of all 17 units-use of primitive contract units helps us interpret the numerical implications of our results. For example, when primitive units are used, our Theorem 8 characterizes the excess stock of goods held by each firm at every stable allocation.

${ }^{10}$ As we point out in Section III.A, the practical implications of our results which involve numerical contract counts are unclear if contracts are not denoted in a fixed unit. Hatfield and Kominers (2010) present an in-depth discussion of these issues.

${ }^{11}$ In our diagrams, an arrow $f_{1} \stackrel{z}{\longrightarrow} f_{2}$ between two firms denotes a contract $z$ with seller $z_{S}=f_{1}$ and buyer $z_{B}=f_{2}$. $f$.

${ }^{12}$ Here, we use the notation $\max _{P^{f}}$ to indicate that the maximization is taken with respect to the preferences of firm
} 


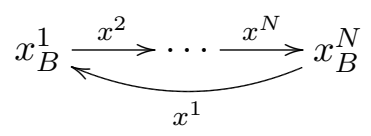

FiguRE 1. A CONTRACT CYCLE.

It will also be convenient to define the choice function for $f$ as a buyer when $f$ has access to the contracts in $Y \subseteq X$ for which $f$ is a buyer and has access to the contracts in $Z \subseteq X$ for which $f$ is a seller. Hence we define

$$
C_{B}^{f}(Y \mid Z) \equiv\left\{x \in C^{f}\left(\left\{y \in Y: y_{B}=f\right\} \cup\left\{z \in Z: z_{S}=f\right\}\right): x_{B}=f\right\} .
$$

Analogously, we define

$$
C_{S}^{f}(Z \mid Y) \equiv\left\{x \in C^{f}\left(\left\{y \in Y: y_{B}=f\right\} \cup\left\{z \in Z: z_{S}=f\right\}\right): x_{S}=f\right\} .
$$

We also define the buyer- and seller-rejected sets as

$$
\begin{aligned}
R_{B}^{f}(Y \mid Z) & \equiv Y-C_{B}^{f}(Y \mid Z), \\
R_{S}^{f}(Z \mid Y) & \equiv Z-C_{S}^{f}(Z \mid Y) .
\end{aligned}
$$

Let $C_{B}(Y \mid Z) \equiv \bigcup_{f \in F} C_{B}^{f}(Y \mid Z)$ be the set of contracts chosen from $Y$ by some firm as a buyer, and $C_{S}(Z \mid Y) \equiv \bigcup_{f \in F} C_{S}^{f}(Z \mid Y)$ be the set of contracts chosen from $Z$ by some firm as a seller. Let $R_{B}(Y \mid Z) \equiv Y-C_{B}(Y \mid Z)$ and $R_{S}(Z \mid Y) \equiv Z-C_{S}(Z \mid Y)$.

An allocation is a set of contracts $A \subseteq X$. Preference relations are extended to allocations in a natural way; for two allocations $W, V \subseteq X$, we write $W \succ_{f} V$ to mean $\left.\left.W\right|_{f} \succ_{f} V\right|_{f}$.

\section{A. Conditions on Preferences}

Full Substitutability. - The primary condition on preferences studied in matching theory is substitutability. Intuitively, contracts $x$ and $y$ are (same-side) substitutes for $f$ if they are the same type of contract for $f$ and they are not complements. For example, if $x_{B}=y_{B}=f$, and $f$ rejects the contract $y$ from $Y$ as a buyer while having access to $Z$ as a seller, $f$ will not choose $y$ from the larger set $\{x\} \cup Y$ while still having access to $Z$ as a seller. The formal definition of same-side substitutability is given below.

DEFINITION 1: The preferences of $f \in F$ are same-side substitutable if for all $Y^{\prime} \subseteq$ $Y \subseteq X$ and $Z^{\prime} \subseteq Z \subseteq X$ 
1) $R_{B}^{f}\left(Y^{\prime} \mid Z\right) \subseteq R_{B}^{f}(Y \mid Z)$ and

2) $R_{S}^{f}\left(Z^{\prime} \mid Y\right) \subseteq R_{S}^{f}(Z \mid Y)$.

Note that this condition is over offer sets; it states that any contract that is rejected from a smaller offer set is also rejected from a larger one.

However, for models where firms can be both buyers and sellers, we must consider how additional offers on one side of the market changes firms' choices on the other side of the market. The key condition here, introduced by Ostrovsky (2008), is crossside complementarity. Intuitively, contracts $y$ and $z$, where $y_{B}=f=z_{S}$, are crossside complements for $f$ if whenever $f$ chooses $y$ from $Y$ as a buyer when the set $Z$ of contracts is available to $f$ as a seller, $f$ still chooses $y$ from $Y$ when $\{z\} \cup Z$ is available to $f$ as a seller.

DEFINITION 2: The preferences of $f \in F$ are cross-side complementary if for all $Y^{\prime} \subseteq Y \subseteq X$ and $Z^{\prime} \subseteq Z \subseteq X$

1) $R_{B}^{f}(Y \mid Z) \subseteq R_{B}^{f}\left(Y \mid Z^{\prime}\right)$ and

2) $R_{S}^{f}(Z \mid Y) \subseteq R_{S}^{f}\left(Z \mid Y^{\prime}\right)$.

Same-side substitutability and cross-side complementarity are closely linked. For illustration, suppose that each contract delineates the transfer of an object from the seller to the buyer. If a firm's preferences are both same-side substitutable and cross-side complementary, then that firm has "substitutable" preferences over objects: The firm is more willing to buy an object if either there are fewer other objects available to buy (same-side substitutes), or there are more opportunities for the firm to sell objects the firm already possesses (cross-side complements). Similarly, the firm is more willing to sell an object if either there are fewer other opportunities to sell objects the firm already possesses (same-side substitutes), or more opportunities to buy an object to replace the one the firm is losing (cross-side complements). In other words, the more objects the firm currently holds, the less willing the firm is to buy/keep new objects. Hence we shall call fully substitutable any preference relation that is both same-side substitutable and cross-side complementary.

We can characterize the set of preferences which are fully substitutable. We describe the set of contracts that $f$ may choose to sign by the offer vector $\mathbf{q}^{f}=\left(\mathbf{q}_{x}^{f}\right)_{\left.x \in X\right|_{f}}$ defined by

$$
\mathbf{q}_{x}^{f}(Y)= \begin{cases}0 & x_{B}=f \text { and } x \in Y \\ -1 & x_{B}=f \text { and } x \notin Y \\ 0 & x_{S}=f \text { and } x \in Y \\ 1 & x_{S}=f \text { and } x \notin Y .\end{cases}
$$

Intuitivively, $\mathbf{q}_{x}^{f}(Y)$ refers to the "object" associated with contract $x$. A value of 1 is given if the firm currently owns the object but cannot sell it. A value of 0 is given if 
either the firm does not currently own the object but may choose to buy it, or the firm currently owns the object but may choose to sell it. Finally, a value of -1 is given if the firm does not currently own the object and can not buy it. Thus $\mathbf{q}_{x}^{f}(Y) \leq \mathbf{q}_{x}^{f}\left(Y^{\prime}\right)$ if and only if $f$ has weakly more buying opportunities, and weakly fewer selling opportunities, in $Y$ than in $Y^{\prime}$.

Using the above notation, we can represent preferences over offer sets with an indirect utility function $u$ over offer vectors. ${ }^{13}$ An indirect utility function $u$ represents a preference relation $P^{f}$ if for all $Y, Y^{\prime} \subseteq X$,

$$
u\left(\mathbf{q}^{f}(Y)\right)>u\left(\mathbf{q}^{f}\left(Y^{\prime}\right)\right) \Leftrightarrow C^{f}(Y) \succ_{f} C^{f}\left(Y^{\prime}\right) \text { under } P^{f} .
$$

Of particular interest are preferences that induce a quasisubmodular indirect utility function. $^{14}$

THEOREM 1: The preferences $P^{f}$ of $f \in F$ are fully substitutable if and only if every indirect utility function representing $P^{f}$ is quasisubmodular.

Submodularity of the indirect utility function is the key condition in demand theory for preferences to be demand-theory substitutable (Ausubel and Milgrom (2002)). However, in the absence of transferable utility it is impossible to quantify the increase in utility from a newly available contract and therefore we can only characterize the utility functions in terms of the ordinal notion of quasisubmodularity. ${ }^{15}$

The Laws of Aggregate Demand and Supply. - A number of important results in two-sided matching theory rely on the Law of Aggregate Demand, which was first introduced by Hatfield and Milgrom (2005). ${ }^{16}$ We generalize this concept to the matching in networks framework with the following definitions.

DEFINITION 3: The preferences of $f$ satisfy the Law of Aggregate Demand if for all $Y, Z \subseteq X$ and $Y^{\prime} \subseteq Y$,

$$
\left|C_{B}^{f}(Y \mid Z)\right|-\left|C_{B}^{f}\left(Y^{\prime} \mid Z\right)\right| \geq\left|C_{S}^{f}(Z \mid Y)\right|-\left|C_{S}^{f}\left(Z \mid Y^{\prime}\right)\right|
$$

\footnotetext{
${ }^{13}$ The utility function is indirect, as it is a function of the contracts available to the firm, as opposed to what contracts the firm actually chooses.

${ }^{14}$ Recall that $u$ is quasisubmodular if for all $\mathbf{q} \leq \mathbf{r}$ and $\mathbf{s} \geq 0$ we have that

$$
\begin{aligned}
& u(\mathbf{r}+\mathbf{s})-u(\mathbf{r})>0 \Rightarrow u(\mathbf{q}+\mathbf{s})-u(\mathbf{q})>0 \\
& u(\mathbf{q}+\mathbf{s})-u(\mathbf{q})<0 \Rightarrow u(\mathbf{r}+\mathbf{s})-u(\mathbf{r})<0
\end{aligned}
$$
}

\footnotetext{
${ }^{15}$ For firms who are either only buyers or only sellers, Hatfield and Kominers (2010) show that if preferences are (same-side) substitutable, then not only is every function that represents these preferences quasisubmodular, but one can always find a submodular function that represents these preferences. This second result relies on a technique introduced by Chambers and Echenique (2009), who show that for any monotone quasisubmodular function there exists a monotonic transformation that transforms that function into a submodular utility function. A similar technique can not be any applied to the current setting, as the indirect utility function is not monotonic in the offer vector.

${ }^{16}$ Alkan and Gale (2003) introduced a related condition called "size monotonocity."
} 
and satisfy the Law of Aggregate Supply if for all $Y, Z \subseteq X$ and $Z^{\prime} \subseteq Z$,

$$
\left|C_{S}^{f}(Z \mid Y)\right|-\left|C_{S}^{f}\left(Z^{\prime} \mid Y\right)\right| \geq\left|C_{B}^{f}(Y \mid Z)\right|-\left|C_{B}^{f}\left(Y \mid Z^{\prime}\right)\right|
$$

Although these conditions are technical to state, their interpetations are straightforward. The Law of Aggregate Demand states that when a firm receives more options as a buyer (while retaining the same options as a seller), its excess stock increases-the number of contracts the firm chooses as a seller does not increase more than the number of contracts the firm chooses as a buyer does. The intuition of the the Law of Aggregate Supply is analogous: under this condition, when a firm receives more options as a seller (while retaining the same options as a buyer), its excess stock decreases.

Note that the Laws of Aggregate Demand and Supply generalize the analogous definitions from two-sided matching. In two-sided matching, if a firm is a buyer, then that firm does not choose any contracts as a seller, and so the right-hand side of the Law of Aggregate Demand vanishes, and hence the Law of Aggregate Demand reduces to its usual statement, that a firm, upon recieving additional offers, chooses at least as many offers as it did before. However, when a firm can be both a buyer and a seller of contracts, the condition is more subtle. The Law of Aggregate Demand now imposes that when a firm obtains additional offers as a buyer, that firm takes on at least as many new contracts as a buyer as the firm takes on as a seller. Intuitively, the condition states that when a firm recieves and accepts a new offer where the firm is a buyer, while holding onto its other buy offers, that firm will then sell at most one new item. Similarly, the Law of Aggregate Supply can be interpeted to say that when the firm receives a new offer to sell that the firm accepts while holding onto its other sell offers, the firm will choose to buy at most one new item.

\section{B. Solution Concepts}

StABILITY. - The key question in matching theory is whether an allocation $A$ is stable, that is, whether there exists a blocking set of contracts $Z$ such that all firms in $Z_{F}$ will choose their contracts in $Z$ from $Z \cup A$ (and possibly drop contracts in $A$ ).

\section{DEFINITION 4: An allocation $A$ is stable if it is}

1) Individually rational: for all $f \in F, C^{f}(A)=\left.A\right|_{f}$;

2) Unblocked: There does not exist a nonempty blocking set $Z \subseteq X$ such that $Z \nsubseteq A$ and for all $f \in Z_{F},\left.Z\right|_{f} \subseteq C^{f}(A \cup Z)$.

Stability is inherently a price-theoretic notion. For an allocation $A$ to be stable, we must be able to find offer sets (that is, sets of contracts offered to each firm) such that

- each contract in $X$ is offered to some firm and 
- each contract not part of the allocation $A$ is rejected by every firm to which it is offered. ${ }^{17}$

These offer sets are similar to price-theoretic budget sets, in which prices define for each agent the set of bundles that are available to that agent. In the context of matching theory, market clearing means that a contract is chosen by its buyer if and only if it is also chosen by its seller. As in price theory, then, a matching-theoretic equilibrium (i.e. a stable allocation) is a set of bundles chosen by each agent (from its offer set) such that the market clears. This is similar to the idea of competitive equilibrium in price theory, where the demand for each item, given the prices, exactly equals the supply. ${ }^{18}$

Our stability notion is the natural generalization of the prior notions of stability in the one-to-one and many-to-one matching literatures. ${ }^{19}$ As we discuss in the next section, this definition of stability is more stringent than the chain stability and tree stability concepts introduced by Ostrovsky (2008).

Stronger notions of stability (which we do not explore) allow for firms to play "strategically," that is, to take on a set of contracts $Z$ from which they obtain a better overall allocation, even though a particular contract $z \in Z$ may not be part of $C^{f}(Z \cup A)$ for some $f$. However, allocations satisfying these stronger notions of stability, such as setwise stability (Sotomayor (1999), Westkamp (2010)) or strong pairwise stability (Echenique and Oviedo (2006)) often do not exist even for reasonable preferences. ${ }^{20}$

Chain Stability. - Ostrovsky (2008) introduced the following notions of chains and chain stability.

DEFINITION 5: A set of contracts $\left\{x^{1}, \ldots, x^{N}\right\}$ is a chain if

1) $x_{B}^{n}=x_{S}^{n+1}$ for all $n=1, \ldots, N-1$,

2) $x_{S}^{n}=x_{S}^{m}$ implies that $n=m$, and

3) $x_{B}^{N} \neq x_{S}^{1}$.

DEFINITION 6: An allocation A is chain stable if it is individually rational and there is no chain that is a blocking set.

Intuitively, contracts form a chain if each firm holding a contract in the chain sells to the next firm in the chain (Condition 1 of Definition 5), the chain never doubles back on

\footnotetext{
${ }^{17}$ Here, we use the terminology "offer set" instead of "choice set" since firms are typically allowed to choose only one option (or point, or bundle) from a choice set. (See the definition given on the first page of Chapter 1 of Mas-Colell, Whinston and Green (1995) for instance.) Here the firms may choose any subset of contracts offered. Formally the choice set is the power set of the offer set.

${ }^{18}$ Hatfield et al. (2011) further develop this idea.

${ }^{19}$ Roth and Sotomayor (1990) and Hatfield and Milgrom (2005) define stability in the one-to-one and many-to-one contexts, respectively. Hatfield and Kominers (2010) discuss the relationship between these concepts.

${ }^{20}$ Additionally, we do not explore the solution concept of the core here, as it is known from an example of Blair (1988) that the core and the set of stable allocations are distinct even in the more restrictive setting of many-to-many matching (without contracts)
} 


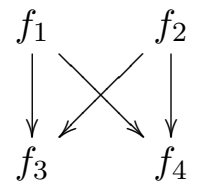

FIGURE 2. A CONTRACT STRUCTURE FOR WHICH CHAIN STABILITY NEED NOT IMPLY STABILITY.

itself (Condition 2 of Definition 5), and the chain is not a loop, i.e. the buyer of the last contract is not the seller of the first (Condition 3 of Definition 5). An allocation is chain stable if it is not blocked by chains of contracts.

Chain stability is not equivalent to the standard notions of stability used in the matching literature, which are special cases of our stability notion (see Roth and Sotomayor (1990)). Rather, chain stability is equivalent to pairwise stability, a much weaker concept. We later show (in Theorem 7) that chain stability is equivalent to stability in the presence of fully substitutable preferences and an acyclic contract set. However, the equivalence only holds when the contract set is acyclic and preferences are fully substitutable. If either condition fails, then the set of stable allocations may be a strict subset of the set of chain stable allocations and, furthermore, chain stability may be an intuitively unappealing solution concept.

For instance, consider the example where $F=\left\{f_{1}, f_{2}, f_{3}, f_{4}\right\}$ and

$$
X=\left\{(i, j): f_{i}, f_{j} \in F \text { and } i \leq 2<j\right\},
$$

as depicted in Figure 2, and suppose firms have the following preferences:

$$
\begin{aligned}
& P^{f_{1}}:\{(1,3)\} \succ\{(1,4)\} \succ \varnothing, \\
& P^{f_{2}}:\{(2,4)\} \succ\{(2,3)\} \succ \varnothing, \\
& P^{f_{3}}:\{(1,3),(2,3)\} \succ \varnothing, \\
& P^{f_{4}}:\{(1,4)\} \succ\{(2,4)\} \succ \varnothing .
\end{aligned}
$$

Note that the preferences of firm $f_{3}$ are not same-side substitutable. There does not exist a stable allocation; however, $\{(1,4)\}$ is chain stable, as the only blocking set is $\{(1,3),(2,3)\}$, which-although seemingly a very natural deviation-is not a chain.

Similarly, when the contract set is not acyclic, it is also easy to construct examples where chain stability is strictly weaker than stability. For instance, consider the following example, where $x_{S}=y_{B}=f$, and $x_{B}=y_{S}=g$ :

$$
\begin{aligned}
& P^{f}:\{x, y\} \succ \varnothing, \\
& P^{g}:\{x, y\} \succ \varnothing .
\end{aligned}
$$


These preferences are fully substitutable; however, the contract set $X$ is not acyclic, as it admits the cycle $\{x, y\}$. For this example, the set $\{x, y\}$ is the only stable allocation, while both $\{x, y\}$ and $\varnothing$ are chain stable. The $\varnothing$ allocation is an unappealing solution to this problem on both normative and positive grounds: $\{x, y\}$ is Pareto preferred to $\varnothing$, and it seems unreasonable to consider a solution concept which does not allow $f$ and $g$ to take part in a joint deviation from $\varnothing$ to $\{x, y\}$.

STRATEGY-PRoOfNESS. - We also consider strategic properties of matching mechanisms. A matching mechanism $\psi$ is a mapping from the set of preference profiles to the set of allocations. In particular, we examine whether certain matching mechanisms are strategy-proof for some firms; that is, whether or not it is a weakly dominant strategy for firms to truthfully reveal their preferences.

DEFINITION 7: A matching mechanism $\psi$ is strategy-proof for $G \subseteq F$ if, for all $g \in G$ and any preference profile $P$, there is no preference profile $\bar{P}^{g}$ such that $\psi\left(\bar{P}^{g}, P^{-g}\right)$ is strictly preferred to $\psi(P)$ by $g .{ }^{21}$

Similarly, we can consider the incentives of groups of firms under a given matching mechanism.

DEFINITION 8: A matching mechanism $\psi$ is group strategy-proof for $G \subseteq F$ if, for all $H \subseteq G$ and any preference profile $P$, there is no preference profile $\bar{P}_{H}$ such that $\psi\left(\bar{P}^{H}, P^{-H}\right)$ is strictly preferred to $\psi(P)$ for all $h \in H$.

As is standard in the matching literature, for a matching mechanism to not be group strategy-proof, the deviation from truth-telling must make all firms in the coalition strictly better off. ${ }^{22}$

\section{Existence of Stable Allocations}

\section{A. Sufficient Conditions for Stability}

In this section, we demonstrate that fully substitutable preferences are sufficient to guarantee the existence of a lattice of stable allocations when the contract set is acyclic, and for the standard opposition of interest results to hold. We give direct proofs of all the results in this section. In Section II.C, we show that these results may be alternatively derived in an indirect fashion: we show (in Theorem 7) that when preferences are fully substitutable and the contract set is acyclic, chain stability and stability are equivalent, hence our existence and opposition results can be derived from the corresponding results of Ostrovsky (2008) for chain stable allocations.

\footnotetext{
${ }^{21}$ Here by $P^{-g}$ we mean the preference profile of all firms excluding $g$. Similarly, $P^{-G}$ is the preference profile of all firms except those in $G \subseteq F$.

${ }^{22}$ Note that this definition is strictly weaker than the definition used in the theory of the allocation of indivisible goods, which allows for deviations for which some members of the coalition are indifferent (Pápai (2000)). Hatfield and Kojima (2009) provide a discussion of the motivation behind this definition.
} 
To prove the existence of a stable allocation, we introduce the operator

$$
\begin{aligned}
\Phi_{B}\left(X^{B}, X^{S}\right) & \equiv X-R_{S}\left(X^{S} \mid X^{B}\right) \\
\Phi_{S}\left(X^{B}, X^{S}\right) & \equiv X-R_{B}\left(X^{B} \mid X^{S}\right) \\
\Phi\left(X^{B}, X^{S}\right) & \equiv\left(\Phi_{B}\left(X^{B}, X^{S}\right), \Phi_{S}\left(X^{B}, X^{S}\right)\right),
\end{aligned}
$$

which generalizes the deferred acceptance algorithm of Gale and Shapley (1962). The inputs $X^{B}$ and $X^{S}$ are sets of contracts which, respectively, contain the options available to the firms as buyers, and the options available to the firms as sellers. At each iteration of $\Phi$, we obtain a new set of seller options which includes all of $X$ except $R_{B}\left(X^{B} \mid X^{S}\right)$, the set of contracts currently available to firms as buyers that they are rejecting. Similarly, the new set of buyer options is all of $X$ except $R_{S}\left(X^{S} \mid X^{B}\right)$.

We first consider fixed points of the operator, and show that these fixed points correspond to stable allocations. Intuitively, at a fixed point we have that every contract is either being accepted (and hence is in both $X^{B}$ and $X^{S}$ ), being rejected by a buyer (and hence is in $X^{B}$ but not $X^{S}$ ) or being rejected by a seller (and hence is in $X^{S}$ but not $X^{B}$ ). Since every contract not in $X^{B} \cap X^{S}$ is being rejected by some firm, there does not exist a blocking set of contracts $Z$ such that each firm will desire all of those contracts, so long as preferences are fully substitutable and the contract set is acyclic.

THEOREM 2: Suppose that the set of contracts $X$ is acyclic and that all firms' preferences are fully substitutable. Then if $\Phi\left(X^{B}, X^{S}\right)=\left(X^{B}, X^{S}\right)$, the allocation $X^{B} \cap X^{S}$ is stable. Conversely, if $A$ is a stable allocation, there exist $X^{B}, X^{S} \subseteq X$ such that $\Phi\left(X^{B}, X^{S}\right)=\left(X^{B}, X^{S}\right)$ and $X^{B} \cap X^{S}=A$.

To see that full substitutablity is necessary for stable allocations to generate fixed points, consider an example where $F=\left\{f_{1}, f_{2}\right\}$ and $X=\{x, y\}$, where $x_{S}=y_{S}=f_{1}$ and $x_{B}=y_{B}=f_{2}$, as shown in Figure 3. Now, consider the following preferences:

$$
\begin{aligned}
& P^{f_{1}}:\{x, y\} \succ \varnothing, \\
& P^{f_{2}}:\{x\} \succ\{y\} \succ \varnothing .
\end{aligned}
$$

Note that the preferences of $f_{1}$ are not fully substitutable. In this example, $\varnothing$ is the unique stable allocation, and yet there do not exist $\left(X^{B}, X^{S}\right)$ such that $X^{B} \cap X^{S}=\varnothing$ and $\Phi\left(X^{B}, X^{S}\right)=\left(X^{B}, X^{S}\right)$. If either $x$ or $y$ is in $X^{B}$, then one of these contracts is not rejected by the buyer $f_{2}$, and hence this contract is in $X^{S}=\Phi_{S}\left(X^{B}, X^{S}\right)=$ $X-R_{B}\left(X^{B} \mid X^{S}\right)$, contradicting the fact that $X^{B} \cap X^{S}=\varnothing$. If both $x$ and $y$ are in $X^{S}$, then neither is rejected by the seller $f_{1}$, and so both are also in $X^{B}=\Phi_{B}\left(X^{B}, X^{S}\right)=$ $X-R_{S}\left(X^{S} \mid X^{B}\right)$, again contradicting the fact that $X^{B} \cap X^{S}=\varnothing$.

To see that full substitutablity is necessary for fixed points to yield stable allocations, 


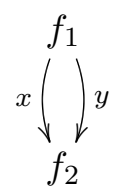

FIGURE 3. A SIMPLE CONTRACT STRUCTURE FOR WHICH THE FIXED-POINT CHARACTERIZATION FAILS IF FIRMS' PREFERENCES ARE NOT FULLY SUBSTITUTABLE.

consider altering the example above so that preferences are given by

$$
\begin{aligned}
& P^{f_{1}}:\{x, y\} \succ \varnothing, \\
& P^{f_{2}}:\{x, y\} \succ \varnothing .
\end{aligned}
$$

In this case, $\{x, y\}$ is the unique stable allocation, but $(\{x\},\{y\})$ is a fixed point and corresponds to the allocation $\varnothing .^{23}$

Furthermore, acyclicity is necessary for fixed points to induce stable allocations. Consider the case where there are three firms, $f_{1}, f_{2}$, and $g$, and the contract structure is as shown in Figure 4. When firms' preferences are given by

$$
\begin{aligned}
P^{f_{1}} & :\left\{y, x^{2}\right\} \succ\left\{x^{1}, x^{2}\right\} \succ \varnothing, \\
P^{f_{2}} & :\left\{x^{2}, x^{1}\right\} \succ \varnothing, \\
P^{g} & :\{y\} \succ \varnothing,
\end{aligned}
$$

no stable allocation exists. However, $\left(X^{B}, X^{S}\right)=\left(\varnothing,\left\{y, x^{1}, x^{2}\right\}\right)$ is a fixed point of the operator $\Phi .{ }^{24}$

Theorem 2 shows that when preferences are fully substitutable and the contract set is acyclic, there is a bijective correspondence between the set of fixed points of $\Phi$ and the set of stable allocations. We now define an order $\sqsubseteq$ on $X \times X$ :

$$
\left(X^{B}, X^{S}\right) \sqsubseteq\left(\bar{X}^{B}, \bar{X}^{S}\right) \text { if } X^{B} \subseteq \bar{X}^{B} \text { and } X^{S} \supseteq \bar{X}^{S} .
$$

\footnotetext{
${ }^{23}$ Observe that $\varnothing$ is chain stable in this example. Thus, note that focusing on stable allocations rather than chain stable allocations allows us to obtain a sharp fixed-point characterization: full substitutability is necessary for both directions of Theorem 2.

${ }^{24}$ It may be the case, even when stable allocations do exist, that fixed points do not correspond to stable allocations. Consider the case where $F=\left\{f_{1}, f_{2}\right\}$ and $X=\{x, y\}$, where $x_{S}=y_{B}=f_{1}$ and $x_{B}=y_{S}=f_{2}$, and preferences are given by:

$$
\begin{aligned}
& P^{f_{1}}:\{x, y\} \succ \varnothing, \\
& P^{f_{2}}:\{x, y\} \succ \varnothing,
\end{aligned}
$$

which are fully substitutable. The allocation $\{x, y\}$ is the unique stable allocation, but $(\{x, y\}, \varnothing)$ is a fixed point of $\Phi$ even though $\varnothing$ is not a stable allocation.
} 


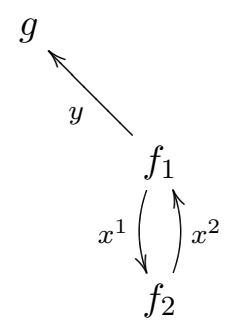

Figure 4. A CONTRACT CYCLE WITH AN OUTSIDE OPTION.

It is clear that $\Phi$ is isotone with respect to this order if preferences are fully substitutable. ${ }^{25}$ Hence, by Tarski's theorem, there exists a lattice of fixed points of the operator $\Phi$. Furthermore, if the contract set is acyclic, then these fixed points correspond to stable allocations.

THEOREM 3: Suppose that the set of contracts $X$ is acyclic and that preferences are fully substitutable. Then there exists a nonempty finite lattice of fixed points $\left(X^{B}, X^{S}\right)$ of $\Phi$ which correspond to stable allocations $A=X^{B} \cap X^{S}$.

Furthermore, the lattice of fixed points has the same structure as in standard bilateral matching contexts. An allocation $\hat{A}$ is the buyer-optimal stable allocation if it preferred to any other stable allocation $A$ by all firms who are exclusively buyers, that is $\hat{A} \succcurlyeq_{g} A$ for any stable allocation $A$ and for all $g \in\left\{f \in F: \nexists x \in X\right.$ such that $\left.x_{S}=f\right\}$. Since the set of the fixed points is a lattice, there exists a highest fixed point with respect to the order $\sqsubseteq$, which we denote by $\left(\hat{X}^{B}, \hat{X}^{S}\right)$. Since this fixed point has the largest set of contracts for the buyers to choose from, it is the unanimously most preferred stable allocation for all firms who are buyers but not sellers. Similarly, the lowest fixed point with respect to $\sqsubseteq$, denoted $\left(\check{X}^{B}, \check{X}^{S}\right)$, is the unanimously most preferred stable allocation for all firms who are sellers but not buyers.

THEOREM 4: Suppose that the set of contracts $X$ is acyclic and that preferences are fully substitutable. Then the highest fixed point $\left(\hat{X}^{B}, \hat{X}^{S}\right)$ of $\Phi$ corresponds to the buyer-optimal stable allocation $\hat{X}^{B} \cap \hat{X}^{S}$, and the lowest fixed-point $\left(\check{X}^{B}, \check{X}^{S}\right)$ of $\Phi$ corresponds to the seller-optimal stable allocation $\check{X}^{B} \cap \check{X}^{S}$.

Building upon machinery developed by Hatfield and Milgrom (2005), our proof of the existence of stable allocations naturally generalizes the deferred acceptance approach

${ }^{25}$ This means that, when preferences are fully substitutable,

$$
\left(X^{B}, X^{S}\right) \sqsubseteq\left(\hat{X}^{B}, \hat{X}^{S}\right) \Rightarrow \Phi\left(X^{B}, X^{S}\right) \sqsubseteq \Phi\left(\hat{X}^{B}, \hat{X}^{S}\right) .
$$


of Gale and Shapley (1962) to the context of matching with networks. Consequently, our existence (Theorem 3) and opposition of interest (Theorem 4) results naturally and directly generalize those of Gale and Shapley (1962) (for one-to-one and many-to-one matching), Roth (1984) (for many-to-many matching), Hatfield and Milgrom (2005) (for many-to-one matching with contracts), and Hatfield and Kominers (2010) (for manyto-many matching with contracts). ${ }^{26}$ Additionally, these results generalize the analogous results due to Echenique and Oviedo (2006) (for many-to-many matching) and Ostrovsky (2008) (for supply-chain matching).

\section{B. Necessary Conditions for Stability}

From the preceding analysis it is clear that, in order to ensure the existence of a stable allocation, it is sufficient that all firms' preferences are fully substitutable and that the set of contracts is acyclic. In many-to-one matching with contracts, however, there exist weaker conditions on preferences that guarantee the existence of a stable allocation. However, for the more general model of matching in networks, both conditions are necessary: If acyclicity fails, then there are fully substitutable preferences for each firm such that no stable allocation exists. For acyclic contract sets, if one firm has preferences that are not fully substitutable, then there exist fully substitutable preferences for the other firms such that no stable allocation exists.

THEOREM 5: If the set of contracts $X$ admits a cycle $L=\left\{x^{1}, \ldots, x^{N}\right\}$ and there exists a firm $g \notin L_{F}$ and a contract between $g$ and some firm in the cycle, then there exist fully substitutable preferences such that no stable allocation exists.

An example of fully substitutable preferences where no stable match exists is given in the discussion of acyclicity in Section II.A. Consider again the contract structure given in Figure 4. Intuitively, if contracts form a cycle (such as that between $f_{1}$ and $f_{2}$ ), then it is easy to construct fully substitutable preferences such that each firm wants to buy if and only if it has an opportunity to sell, and wants to sell if and only if it has the opporutunity to buy. With these preferences, the empty allocation is not stable, as all members of the cycle agree that the contract cycle is better than nothing. However, if one member of the cycle $\left(f_{1}\right)$ has an outside option as a buyer $(y)$, then the cycle itself may not be stable as that one member may most prefer to choose this outside option as a buyer while keeping the contract where the firm is a seller $\left(x^{2}\right)$ from the cycle. This implies that the cycle itself is unstable. However, any other allocation (such as $\left\{y, x^{2}\right\}$ ) is also unstable, as it is not individually rational for some firm. Hence, we see that even when preferences are reasonable, contract sets containing cycles may not admit stable allocations.

Furthermore, it is also necessary that the preferences of each firm be fully substitutable. Consider again the example from Section I.B (pictured in Figure 2), where $F=\left\{f_{1}, f_{2}, f_{3}, f_{4}\right\}$ and $X=\left\{(i, j): f_{i}, f_{j} \in F\right.$ and $\left.i \leq 2<j\right\}$. Here, firms $f_{1}$ and

\footnotetext{
${ }^{26}$ Note that a stronger "polarization of interests" result (such as that of Theorem 2.13 of Roth and Sotomayor (1990)) is ruled out in our setting by an example of Konishi and Ünver (2006).
} 
$f_{2}$ are sellers, and firms $f_{3}$ and $f_{4}$ are buyers. Suppose that preferences are given by:

$$
\begin{aligned}
& P^{f_{1}}:\{(1,3)\} \succ\{(1,4)\} \succ \varnothing, \\
& P^{f_{2}}:\{(2,4)\} \succ\{(2,3)\} \succ \varnothing, \\
& P^{f_{3}}:\{(1,3),(2,3)\} \succ \varnothing, \\
& P^{f_{4}}:\{(1,4)\} \succ\{(2,4)\} \succ \varnothing .
\end{aligned}
$$

It is not stable for both sellers $f_{1}$ and $f_{2}$ to sell to $f_{3}$, as then $f_{2}$ would like to deviate and sell to $f_{4}$, and $f_{4}$ would like to buy from $f_{2}$. That, however, is also not stable, as then $f_{3}$ would buy nothing, and so $f_{1}$ would then sell to $f_{4}$. This is also not stable, as then both sellers would prefer to sell to $f_{3}$. Hence, no allocation is individually rational and unblocked. Generalizing this example, we obtain the following theorem.

THEOREM 6: Suppose $X$ is exhaustive and acyclic, and there exists a firm $f$ whose preferences are not fully substitutable, and there exist at least two firms upstream of $f$ and two firms downstream of $f$. Then there exist fully substitutable preferences for the firms other than $f$ such that no stable allocation exists. ${ }^{27}$

This result is suprising, as no analogous result holds in the Hatfield and Milgrom (2005) setting of many-to-one matching with contracts (Hatfield and Kojima (2008)). ${ }^{28}$ Indeed, Hatfield and Kojima (2010) and Hatfield and Kominers (2010) have found conditions on preferences weaker than substitutability that guarantee the existence of a stable allocation in the setting of many-to-one matching with contracts. The key distinction between our setting and that of Hatfield and Milgrom (2005) is that in our setting, all firms may demand multiple contracts as both buyers and sellers; this fact is crucial to the proof of Theorem 6 .

\section{The Relationship between Stability and Chain Stability}

It is immediate that, regardless of restrictions on preferences or the contract set, any stable allocation is chain stable. ${ }^{29}$ However, as we now show, stability and chain stability coinicide on domains where $X$ is acyclic and preferences are fully substitutable.

THEOREM 7: Suppose that the set of contracts $X$ is acyclic and that all firms' preferences are fully substitutable. Then an allocation $A$ is stable if and only if it is chain stable.

Ostrovsky (2008) proved, under the assumption of fully substitutable preferences, that chain stability is equivalent to an alternative solution concept called tree stability. ${ }^{30}$ Like

\footnotetext{
${ }^{27}$ Slightly weaker conditions on the number of firms other than $f$ can be stated. However, these conditions are exceedingly technical; see the proof of Theorem 6 for details.

${ }^{28}$ Confusingly, Hatfield and Milgrom (2005) claimed such a result, but this claim was later found to be in error (Hatfield and Kojima (2008)).

${ }^{29}$ This follows as chain stability requires that blocking sets be chains, whereas our notion of stability puts no restrictions on the structure of the blocking set.

${ }^{30}$ See Theorem 4 of Ostrovsky (2008).
} 
chain stability, tree stability is weaker than stability, in general. However, stability and tree stability are equivalent under fully substitutable preferences; this fact is a simple corollary of Theorem 4 of Ostrovsky (2008) and our Theorem 7.

It follows from the preceding discussion that our Theorems 2-4 can be obtained indirectly using Theorem 7 and the Ostrovsky (2008) fixed-point characterization, existence, and opposition of interest results. However, there is no necessity result for chain stability corresponding to our Theorem 6 . To see this, consider a firm $f$ with preferences of the form

$$
P^{f}:\{x, y\} \succ \varnothing
$$

where $x_{B}=y_{B}=f$, which are not fully substitutable. A chain stable allocation exists for any fully substitutable preferences for other firms so long as the contract set $X$ is acyclic. These chain stable allocations can be found by using the isotone operator $\Phi$ introduced above on the set $X-\{x, y\}$; these allocations will be chain stable as there are (by Theorem 2) no blocking sets not involving firm $f$, and the only blocking set involving firm $f$ possible is $\{x, y\}$, which is not a chain. However, as we discussed in Section I.B, $\{x, y\}$ may be a very natural block.

\section{The Structure of the Set of Stable Allocations}

\section{A. The Rural Hospitals Theorem}

In the celebrated rural hospitals theorem, Roth (1986) proved that in a many-to-one (doctor-hospital) matching market with responsive preferences, any hospital that has unfilled positions at some stable matching is assigned exactly the same set of doctors at every stable matching. ${ }^{31}$ In the context of many-to-one (Hatfield and Milgrom (2005); Hatfield and Kojima (2010)) and many-to-many matching with contracts (Hatfield and Kominers (2010)), the rural hospitals theorem has been (partially) generalized to a statement regarding the number of contracts: in a many-to-many matching market with contracts, if preferences are substitutable and satisfy the Law of Aggregate Demand, then every firm holds the same number of contracts in each stable allocation. ${ }^{32}$ However, this statement is false in the context of matching in networks.

For example, consider the following simple set of preferences, where $F=\left\{f_{1}, f_{2}, f_{3}\right\}$

\footnotetext{
${ }^{31}$ Recall that hospital preferences are called responsive if they are consistent with a complete strict order over individual doctors.

${ }^{32}$ Generalizations of the Roth (1986) rural hospitals theorem to the theory of matching with contracts have focused only upon the total number of contracts signed by each hospital $h$, since in general both the set of doctors with whom $h$ contracts and the contract terms to which $h$ agrees may vary across stable allocations. Indeed, Hatfield and Kojima (2010) give (in their Footnote 21) a many-to-one matching with contracts example in which there is a hospital $h$ with unfilled positions such that both

- the set of doctors $h$ contracts with, and

- the contract terms $h$ receives from doctors
}

vary across stable allocations. Hence, the invariance of the number of contracts per firm across stable allocations seems to be the sharpest possible matching with contracts generalization of the Roth (1986) theorem. 


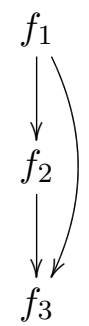

Figure 5. A CONTRACT STRUCTURE IN WHICH THE MOST IMMEDiATE (PUTATIVE) GENERALIZATION OF THE RURAL HOSPITALS THEOREM NEED NOT HOLD.

and $X=\left\{(i, j): f_{i}, f_{j} \in F\right.$ and $\left.i<j\right\}$, as pictured in Figure 5:

$$
\begin{aligned}
& P^{f_{1}}:\{(1,2)\} \succ\{(1,3)\} \succ \varnothing, \\
& P^{f_{2}}:\{(1,2),(2,3)\} \succ \varnothing, \\
& P^{f_{3}}:\{(1,3)\} \succ\{(2,3)\} \succ \varnothing .
\end{aligned}
$$

Note that firm $f_{2}$ has the simplest possible fully substitutable preferences where the set of contracts that firm $f_{2}$ signs as a buyer truly depends on the set of contracts she has access to as a seller. Furthermore, these preferences satisfy the Laws of Aggregate Demand and Supply. However, both $\{(1,3)\}$ and $\{(1,2),(2,3)\}$ are stable allocations, and so we see that the number of contracts signed may vary across stable allocations even when preferences satisfy the Laws of Aggregate Demand and Supply. Rather, the difference for each firm between the number of "buy" and "sell" contracts held is constant across the two stable allocations; it turns out this "balancing" property holds in general.

THEOREM 8: Suppose that the set of contracts $X$ is acyclic and that all firms' preferences are fully substitutable and satisfy the Laws of Aggregate Demand and Supply. Then, for each firm, the difference between the number of contracts that firm buys and the number of contracts that firm sells is invariant across stable allocations.

When all contracts are denoted in a fixed unit (as in exchange economies), Theorem 8 implies that each firm holds the same excess stock at every stable allocation. When contracts do not use a constant unit (as, for example, in labor markets in which both fulland part-time job contracts are available), the exact numerical implications of Theorem 8 are less clear, as Hatfield and Kominers (2010) discuss. However, even in this case, the rural hospitals result is crucial for the strategy-proofness results of Section III.B.

Note that Theorem 8 does generalize the prior rural hospitals theorems: in previous two-sided matching models, a firm $f$ either only buys or only sells, hence Theorem 8 implies that $f$ signs exactly the same number of contracts in every stable allocation. The 
net position in contracts of a firm which both buys and sells is invariant across stable allocations, but the total number of contracts such a firm signs can vary across stable allocations.

Furthermore, the Laws of Aggregate Demand and Supply are the weakest possible conditions that ensure this additional structure on the set of stable allocations.

THEOREM 9: Suppose that the set of contracts $X$ is acyclic and exhaustive. If the preferences of some firm $f$ fail to satisfy either the Law of Aggregate Demand or the Law of Aggregate Supply but are fully substitutable, then there exist fully substitutable preferences for the other firms satisfying the Laws of Aggregate Demand and Supply such that there exist two stable allocations across which the difference between the number of contracts $f$ buys and the number of contracts $f$ sells varies.

\section{B. Strategy-Proofness}

In many-to-one matching with contracts, substitutability and the Law of Aggregate Demand are enough to ensure that the buyer-optimal stable mechanism is strategy-proof for unit demand buyers, i.e. buyers who demand at most one contract. Unfortunately, in many-to-many matching contexts, it is not a weakly dominant strategy for either side of the market to reveal its preferences truthfully to any mechanism which chooses a stable allocation. However, even in the matching in networks framework, if some subset of firms acts only as buyers, and each of these buyers has unit demand, then the mechanism which chooses the buyer-optimal stable allocation will be strategy-proof for these buyers. ${ }^{33}$

THEOREM 10: Suppose that the set of contracts $X$ is acyclic and that firms' preferences are fully substitutable and satisfy the Laws of Aggregate Demand and Supply. If additionally, for all $g \in G \subseteq F$, the preferences of $g$ exhibit unit demand, then any mechanism that selects the buyer-optimal stable allocation is (group) strategy-proof for $G$.

Theorem 10 generalizes results of Dubins and Freedman (1981), Abdulkadiroğlu (2005), Hatfield and Milgrom (2005), and Hatfield and Kojima (2009); its proof follows exactly as in that of Hatfield and Kojima (2009). This result can be used to show a common corollary that the set of unit-demand buyers weakly prefers the buyer-optimal stable allocation to all other individually rational allocations.

COROLLARY 1: Suppose that the set of contracts $X$ is acyclic and that firms' preferences are fully substitutable and satisfy the Laws of Aggregate Demand and Supply. If additionally, for all $g \in G \subseteq F$, the preferences of $g$ exhibit unit demand, then there does not exist an individually rational allocation that every member of $G$ strictly prefers to the buyer-optimal stable allocation. ${ }^{34}$

\footnotetext{
${ }^{33}$ By symmetry, an analogous result applies to sellers.

${ }^{34}$ As with Theorem 10, the proof of Corollary 1 follows as in that of Hatfield and Kojima (2009).
} 


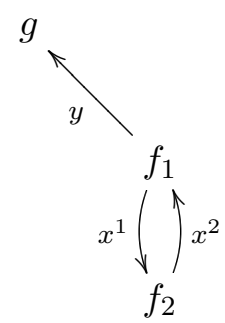

Figure 4. A CONTRACT CYCLE WITH AN OUTSIDE OPTION.

This result is commonly called "weak Pareto optimality" and was first shown by Roth (1982) for one-to-one matching; the most general previous result of this form is for many-to-one matching with contracts (Hatfield and Kojima (2009); Kojima (2007)).

\section{Conclusion}

In this paper we have extended the model of classical matching theory to consider networks of contracts. We have shown that, on the one hand, if the set of contracts is acyclic, and preferences are fully substitutable, not only do stable allocations exist, but they form a lattice. Moreover, classical results of matching theory, such as the rural hospitals and strategy-proofness theorems, generalize to this setting. On the other hand, Theorem 5 shows that, in the presence of fully substitutable preferences over contracts, stability cannot be guaranteed if there is a single cycle in the set of contracts; furthermore, Theorem 6 shows that if even one firm does not have fully substitutable preferences, stability again cannot be guaranteed. Hence our work delineates a strict frontier for classical matching theory, in the sense that both acyclity and full substitutability are both sufficient and necessary for classical matching theory approaches.

However, supplementing the set of contracts with a numeraire (over which utility is quasilinear) may allow us to go further. Consider the simplest example where a cyclic contract set confounds stability, drawn from the proof of Theorem 5. There are three firms, $f_{1}, f_{2}, g$, and the contract structure is as shown in Figure 4, which we reproduce here. When firms' preferences are given by

$$
\begin{aligned}
P^{f_{1}} & :\left\{y, x^{2}\right\} \succ\left\{x^{1}, x^{2}\right\} \succ \varnothing, \\
P^{f_{2}} & :\left\{x^{2}, x^{1}\right\} \succ \varnothing, \\
P^{g} & :\{y\} \succ \varnothing,
\end{aligned}
$$

there exists no stable allocation. Indeed, firm $f_{1}$ prefers its outside option $y$ over $x^{1}$, and hence the trade cycle $\left\{x^{1}, x^{2}\right\}$ always breaks down.

Problems of this form often arise in contracting relationships, and they have a well- 
known solution, albeit one outside the scope of classical matching theory. One resolution to this dilemma dates back to ideas of Vickrey (1961) and Pigou: firm $f_{1}$ should pay a transfer to $f_{2}$ equal to the value of the externality $f_{1}$ causes by dropping contract $x^{1}$ in favor of $y$. Generalizing this intuition, Hatfield et al. (2011) find that transferable utility promotes stability in some new settings.

Even with transferable utility, full substitutability is necessary in order to guarantee the existence of stable allocations (Hatfield et al. (2011)). However, many problems naturally generate complementarities; a hospital may open a new wing only if it acquires doctors of multiple specialities, or a firm may be able to operate more efficiently with more units. ${ }^{35}$ Much work remains to be done to understand the dynamics and equilibria of matching markets with complementarities; we leave this topic for future research.

\footnotetext{
${ }^{35}$ Milgrom (2007) and Day and Milgrom (2008) discuss some of the issues that arise with complementary preferences in package auctions. Klaus and Klijn (2005) discuss the "couples problem," a type of complementary preferences that commonly arises in bilateral matching contexts.
} 


\section{REFERENCES}

Abdulkadiroğlu, Atila. 2005. "College Admissions with Affirmative Action." International Journal of Game Theory, 33: 535-549.

Abdulkadiroğlu, Atila, Parag A. Pathak, Alvin E. Roth, and Tayfun Sönmez. 2005. "The Boston public school match." American Economic Review, 95: 368-371.

Abdulkadiroğlu, Atila, Parag A. Pathak, and Alvin E. Roth. 2005. "The New York City high school match.” American Economic Review, 95: 364-367.

Abdulkadiroğlu, Atila, Parag A. Pathak, and Alvin E. Roth. 2009. "Strategyproofness versus Efficiency in Matching with Indifferences: Redesigning the NYC High School Match." American Economic Review, 99(5): 1954-1978.

Alkan, Ahmet, and David Gale. 2003. "Stable Schedule Matching under Revealed Preference.” Journal of Economic Theory, 112: 289-306.

Ausubel, Larry M., and Paul Milgrom. 2002. "Ascending auctions with package bidding." Frontiers of Theoretical Economics, 1: 1-42.

Blair, Charles. 1988. "The lattice structure of the set of stable matchings with multiple partners." Mathematics of Operations Research, 13: 619-628.

Chambers, Christopher P., and Federico Echenique. 2009. "Supermodularity and preferences." Journal of Economic Theory, 144: 1004-1014.

Crawford, Vincent P., and Elsie Marie Knoer. 1981. "Job Matching with Heterogeneous Firms and Workers." Econometrica, 49(2): 437-450.

Day, Robert, and Paul Milgrom. 2008. "Core-selecting auctions." International Journal of Game Theory, 36: 393-407.

Dubins, L. E., and D. A. Freedman. 1981. "Machiavelli and the Gale-Shapley Algorithm." American Mathematical Monthly, 88: 485-494.

Echenique, Federico, and Jorge Oviedo. 2006. "A theory of stability in many-to-many matching markets." Theoretical Economics, 1: 233-273.

Fox, Jeremy T. 2010. "Identification in matching games." Quantitative Economics, 1: 203-254.

Gale, David, and Lloyd S. Shapley. 1962. "College admissions and the stability of marriage." American Mathematical Monthly, 69: 9-15.

Hatfield, John William, and Fuhito Kojima. 2008. "Matching with Contracts: Comment." American Economic Review, 98: 1189-1194.

Hatfield, John William, and Fuhito Kojima. 2009. "Group Incentive Compatibility for Matching with Contracts." Games and Economic Behavior, 67: 745-749. 
Hatfield, John William, and Fuhito Kojima. 2010. "Substitutes and Stability for Matching with Contracts." Journal of Economic Theory, 145(5): 1704-1723.

Hatfield, John William, and Paul Milgrom. 2005. "Matching with Contracts." American Economic Review, 95: 913-935.

Hatfield, John William, and Scott Duke Kominers. 2010. "Contract Design and Stability in Matching Markets." Mimeo, Harvard Business School.

Hatfield, John William, Scott Duke Kominers, Alexandru Nichifor, Michael Ostrovsky, and Alexander Westkamp. 2011. "Stability and Competitive Equilibrium in Trading Networks." Mimeo, Stanford Graduate School of Business.

Kelso, Alexander S., and Vincent P. Crawford. 1982. "Job Matching, Coalition Formation, and Gross Substitutes." Econometrica, 50: 1483-1504.

Klaus, Bettina, and Flip Klijn. 2005. "Stable Matchings and Preferences of Couples." Journal of Economic Theory, 121: 75-106.

Klaus, Bettina, and Markus Walzl. 2009. "Stable Many-to-Many Matchings with Contracts." Journal of Mathematical Economics, 45(7-8): 422-434.

Kojima, Fuhito. 2007. "The law of aggregate demand and welfare in the two-sided matching market." Economics Letters, 99: 581-584.

Konishi, Hideo, and M. Utku Ünver. 2006. "Credible group-stability in many-to-many matching problems." Journal of Economic Theory, 129: 57-80.

Mas-Colell, Andreu, Michael D. Whinston, and Jerry R. Green. 1995. Microeconomic Theory. Oxford University Press.

Milgrom, Paul. 2004. Putting Auction Theory to Work. Cambridge University Press.

Milgrom, Paul. 2007. "Package auctions and exchanges." Econometrica, 75: 935-965.

Ostrovsky, Michael. 2008. "Stability in supply chain networks." American Economic Review, 98: 897-923.

Pápai, Szilvia. 2000. “Strategyproof assignment by hierarchical exchange.” Econometrica, 68(6): 1403-1433.

Roth, Alvin E. 1982. "The economics of matching: Stability and incentives." Mathematics of Operations Research, 7: 617-628.

Roth, Alvin E. 1984. "Stability and polarization of interests in job matching." Econometrica, 52: 47-57.

Roth, Alvin E. 1985. "The College Admissions Problem is not Equivalent to the Marriage Problem.” Journal of Economic Theory, 36: 277-288. 
Roth, Alvin E. 1986. "On the Allocation of Residents to Rural Hospitals: A General Property of Two-Sided Matching Markets." Econometrica, 54: 425-427.

Roth, Alvin E. 2008. "Deferred acceptance algorithms: history, theory, practice, and open questions.” International Journal of Game Theory, 36: 537-569.

Roth, Alvin E., and Elliott Peranson. 1999. "The effects of the change in the NRMP matching algorithm.” American Economic Review, 89: 748-780.

Roth, Alvin E., and Marilda A. O. Sotomayor. 1990. Two-sided matching: a study in game-theoretic modeling and analysis. Cambridge University Press.

Sotomayor, Marilda A. O. 1999. "Three remarks on the many-to-many stable matching problem." Mathematical Social Sciences, 38: 55-70.

Sotomayor, Marilda A. O. 2004. "Implementation in the many-to-many matching market." Games and Economic Behavior, 46: 199-212.

Vickrey, William. 1961. "Counterspeculation, Auctions and Competitive Sealed Tenders." Journal of Finance, 16: 8-37.

Westkamp, Alexander. 2010. "Market Structure and Matching with Contracts." Journal of Economic Theory, 145(5): 1724-1738. 


\section{Appendix}

\section{Proof of Theorem 1}

Proof OF THE "ONLY IF" DiReCTION. - First, note that it suffices to show the quasisubmodularity conditions hold for the case of adding (or removing) a single contract to two sets $Y, Z \subseteq X$, since if quasisubmodularity fails for some $\mathbf{s} \geq 0$, it must fail for some $\mathbf{r}$ such that $\mathbf{r}_{w} \neq 0$ for only one contract.

Fix some firm $f \in F$, consider any two offer vectors $\mathbf{q}^{f}(Y)$ and $\mathbf{q}^{f}(Z)$ such that $\mathbf{q}^{f}(Y) \leq \mathbf{q}^{f}(Z)$, and suppose that $\mathbf{q}_{w}^{f}(Y)=\mathbf{q}_{w}^{f}(Z)=-1$ for some $w$ such that $w_{B}=f$. Suppose that

$$
u\left(\mathbf{q}^{f}(\{w\} \cup Z)\right)-u\left(\mathbf{q}^{f}(Z)\right)>0 .
$$

Then $w \in C^{f}(\{w\} \cup Z)$, so $w \in C^{f}(\{w\} \cup Y)$ by full substitutability. Hence,

$$
u\left(\mathbf{q}^{f}(\{w\} \cup Y)\right)-u\left(\mathbf{q}^{f}(Y)\right)>0 .
$$

and quasisubmodularity is satisfied. Hence, when we add any contract to the offer set as a buyer, quasisubmodularity is satisfied.

Now suppose that $\mathbf{q}_{w}^{f}(Y)=\mathbf{q}_{w}^{f}(Z)=0$ for some $w$ such that $w_{S}=f$. Suppose that

$$
u\left(\mathbf{q}^{f}(Y-\{w\})\right)-u\left(\mathbf{q}^{f}(Y)\right)<0
$$

then $w \in C^{f}(Y)$, so $w \in C^{f}(Z)$ by full substitutability. Hence,

$$
u\left(\mathbf{q}^{f}(Z-\{w\})\right)-u\left(\mathbf{q}^{f}(Z)\right)<0
$$

and quasisubmodularity is satisfied when we remove any contract to the offer set as a seller.

Finally, note that it is enough to show it for adding (or removing) a single contract to $Y^{q}$ and $Y^{r}$, since if quasisubmodularity fails for some $\mathbf{s} \geq 0$, it must fail for $\mathbf{s}^{\prime}$ such that $\mathbf{s}_{w}^{\prime} \neq 0$ for only one contract.

Proof OF THE "IF" Direction. - Suppose that the preferences of $f \in F$ violate the first condition of same-side substitutability. Then, there exist contracts $x, w \in X$ and $Y \subseteq X$ such that $x_{B}=w_{B}=f$ and

$w \notin C^{f}(Y \cup\{w\})$ and $w \in C^{f}(\{x\} \cup Y \cup\{w\})$. 
It follows that for any indirect utility function $u$ which represents these preferences, we have

$u\left(\mathbf{q}^{f}(Y \cup\{w\})\right)-u\left(\mathbf{q}^{f}(Y)\right)=0<u\left(\mathbf{q}^{f}(\{x\} \cup Y \cup\{w\})\right)-u\left(\mathbf{q}^{f}(\{x\} \cup Y)\right)$.

Thus, $u$ is not quasisubmodular.

Similarly, suppose that the preferences of $f \in F$ violate the second condition of sameside substitutability. Then there exist contracts $x, w \in X$ and $Y \subseteq X$ such that $x_{S}=$ $w_{S}=f$ and

$$
w \notin C^{f}(Y \cup\{w\}) \text { and } w \in C^{f}(\{x\} \cup Y \cup\{w\}) .
$$

It follows that for any indirect utility function $u$ which represents these preferences, we have

$u\left(\mathbf{q}^{f}(Y \cup\{w\})\right)-u\left(\mathbf{q}^{f}(Y)\right)=0<u\left(\mathbf{q}^{f}(\{x\} \cup Y \cup\{w\})\right)-u\left(\mathbf{q}^{f}(\{x\} \cup Y)\right)$.

Thus, $u$ is not quasisubmodular.

Now suppose that the preferences of $f \in F$ violate the first condition of cross-side complementarity. Then there exist contracts $x, w \in X$ and $Y \subseteq X$ such that $x_{S}=$ $w_{B}=f$ such that

$$
w \in C^{f}(Y \cup\{w\}) \text { and } w \notin C^{f}(\{x\} \cup Y \cup\{w\})
$$

It follows that for any indirect utility function $u$ which represents these preferences, we have

$u\left(\mathbf{q}^{f}(\{x\} \cup Y \cup\{w\})\right)-u\left(\mathbf{q}^{f}(\{x\} \cup Y)\right)=0<u\left(\mathbf{q}^{f}(Y \cup\{w\})\right)-u\left(\mathbf{q}^{f}(Y)\right)$.

Thus, $u$ is not quasisubmodular.

Similarly, suppose that the preferences of $f \in F$ violate the second condition of crossside complementarity. Then there exists contracts $x, w \in X$ and $Y \subseteq X$ such that $x_{B}=w_{S}=f$ such that

$$
w \in C^{f}(Y \cup\{w\}) \text { and } w \notin C^{f}(\{x\} \cup Y \cup\{w\}) .
$$

It follows that for any indirect utility function $u$ which represents these preferences, we have

$u\left(\mathbf{q}^{f}(\{x\} \cup Y \cup\{w\})\right)-u\left(\mathbf{q}^{f}(\{x\} \cup Y)\right)=0<u\left(\mathbf{q}^{f}(Y \cup\{w\})\right)-u\left(\mathbf{q}^{f}(Y)\right)$.

Thus, $u$ is not quasisubmodular. 


\section{Proof of Theorem 2}

Since the contractual set $X$ is acyclic, there is an ordering $\triangleleft$ on firms such that $x_{S} \triangleleft$ $x_{B}$ for all $x \in X$. Fix some labeling of firms $f_{1}, \ldots, f_{N}$ so that $f_{n} \triangleleft f_{n+1}$ for all $n=1, \ldots, N-1$.

First Part. - Suppose that $X^{B} \cap X^{S} \equiv A$ is a fixed point, but that $A$ is not stable. Then either $A$ is not individually rational or $A$ admits a blocking set $Z$.

If $A$ is not individually rational, there must exist $x \in A$ such that $x \in R^{f}(A)$ for some $f \in F$. Then either $x \in R_{B}^{f}(A \mid A)$ and $x_{B}=f$ or $x \in R_{S}^{f}(A \mid A)$ and $x_{S}=f$. Assume the former. (The latter case is symmetric.) Then $x \in R_{B}^{f}\left(X^{B} \mid A\right)$ by same-side substitutability. However, every contract in the set $X^{S}-A$ is rejected by some firm as a seller, and so $R_{B}^{f}\left(X^{B} \mid A\right)=R_{B}^{f}\left(X^{B} \mid X^{S}\right)$. Hence $x \in R_{B}^{f}\left(X^{B} \mid X^{S}\right)$, and hence $x \notin X^{S}=\Phi_{S}\left(X^{B}, X^{S}\right)=X-R_{B}\left(X^{B} \mid X^{S}\right)$, and hence $x \notin X^{B} \cap X^{S}=A$, a contradiction.

If there exists a blocking set $Z$ for $A$, consider a contract $z$ such that $z_{S} \unlhd y_{S}$ for all other $y \in Z{ }^{36}$ By same-side substitutability, since $z \in C_{S}^{z_{S}}(Z \cup A \mid Z \cup A)$, we have that $z \in C_{S}^{z_{S}}(\{z\} \cup A \mid Z \cup A)=C_{S}^{z_{S}}(\{z\} \cup A \mid A)$ as there are no contracts in $Z$ such that $z_{S}$ is a buyer by assumption. Hence $z \in C_{S}^{z_{S}}\left(\{z\} \cup X^{S} \mid A\right)$ and then by crossside complementarity, $z \in C_{S}^{z_{S}}\left(\{z\} \cup X^{S} \mid X^{B}\right)$. Hence, if $z \in X^{S}$, then $z \in X^{B}=$ $\Phi_{B}\left(X^{B}, X^{S}\right)=X-R_{S}\left(X^{S} \mid X^{B}\right)$. But $z \notin A=X^{B} \cap X^{S}$ by assumption, and $X^{B} \cup$ $X^{S}=X$, and so $z \in X^{B}$. Now consider an arbitrary contract $w \in Z$, and suppose that for all contracts $y \in Z$ such that $y_{S} \triangleleft w_{S}, y \in X^{B}$. By same-side substitutability, since $w \in C_{S}^{w_{S}}(Z \cup A \mid Z \cup A)$, we have that $w \in C_{S}^{w_{S}}(\{w\} \cup A \mid Z \cup A)$. Now, by induction, for any contract $y \in Z$ such that $y_{B}=w_{S}, y \in X^{B}$. Hence, $\left\{y \in Z: y_{B}=w_{S}\right\} \subseteq X^{B}$, and $A \subseteq X^{B}$, implying that $\left\{y \in Z: y_{B}=w_{S}\right\} \cup A \subseteq X^{B}$, and so by cross-side complemenarity, $w \in C_{S}^{w_{S}}\left(\{w\} \cup A \mid X^{B}\right)$. Therefore $w \in C_{S}^{w_{S}}\left(\{w\} \cup X^{S} \mid X^{B}\right)$. Thus, if $w \in X^{S}$, then $w \in X^{B}=\Phi_{B}\left(X^{B}, X^{S}\right)=X-R_{S}\left(X^{S} \mid X^{B}\right)$. But $w \notin$ $A=X^{B} \cap X^{S}$ by assumption, and $X^{B} \cup X^{S}=X$, and so $w \in X^{B}$. Using induction then, we have that $Z \subseteq X^{B}$. Working symmetrically for buyers, we have that $Z \subseteq X^{S}$. Hence, $Z \subseteq X^{S} \cap X^{\bar{B}}=A$ and hence $Z$ is not a blocking set, a contradiction.

Second Part. - Suppose that $A$ is a stable allocation. We construct $X^{B}$ and $X^{S}$ iteratively over firms. Let $X^{B}(0) \equiv X^{S}(0) \equiv A$. Let

$$
\begin{aligned}
& X^{B}(n) \equiv\left\{x \in\left(X-X^{S}(n-1)\right): x_{B}=f_{n}\right\} \cup X^{B}(n-1) \\
& X^{S}(n) \equiv\left\{x \in X: x \in R_{S}^{f_{n}}\left(\{x\} \cup A \mid X^{B}(n)\right)\right\} \cup X^{S}(n-1) .
\end{aligned}
$$

Finally, let $X^{B}=X^{B}(N)$ and $X^{S}=X^{S}(N)$.

\footnotetext{
${ }^{36}$ Recall that acyclicity guarantees there exists an order $\triangleleft$ on $F$ such that $x_{S} \triangleleft x_{B}$ for all $x \in X$.
} 
We now show that $\left(X^{B}, X^{S}\right)$ is a fixed point. We have

$$
\begin{aligned}
X-R_{S}\left(X^{S} \mid X^{B}\right) & =X-\left(X^{S}-C_{S}\left(X^{S} \mid X^{B}\right)\right) \\
& =X-\bigcup_{n=1}^{N} R_{S}^{f_{n}}\left(\left(X^{S} \mid f_{n}\right) \mid\left(X^{B} \mid f_{n}\right)\right) \\
& =X-\bigcup_{n=1}^{N} R_{S}^{f_{n}}\left(\left(\left.X^{S}(n)\right|_{f_{n}}\right) \mid\left(X^{B} \mid f_{n}\right)\right) \text { as }\left.X^{S}(n)\right|_{f_{n}}=\left.X^{S}\right|_{f_{n}} \\
& =X-\left.\bigcup_{n=1}^{N}\left(X^{S}(n)-A\right)\right|_{f_{n}} \text { as } f_{n} \text { has fully substitutable preferences } \\
& =X-\left(X^{S}-A\right) \text { by the definition of } X^{S} \\
& =X^{B} \text { by the definition of } X^{B} .
\end{aligned}
$$

Similarly, we have

$$
\begin{aligned}
X-R_{B}\left(X^{B} \mid X^{S}\right) & =X-\left(X^{B}-C_{B}\left(X^{B} \mid X^{S}\right)\right) \\
& =X-\bigcup_{n=1}^{N} R_{B}^{f_{n}}\left(\left(\left.X^{B}\right|_{f_{n}}\right) \mid\left(\left.X^{S}\right|_{f_{n}}\right)\right) \\
& =X-\bigcup_{n=1}^{N} R_{B}^{f_{n}}\left(\left(\left.X^{B}(n)\right|_{f_{n}}\right) \mid\left(\left.X^{S}\right|_{f_{n}}\right)\right) \text { as }\left.X^{B}(n)\right|_{f_{n}}=\left.X^{B}\right|_{f_{n}} \\
& =X-\left.\bigcup_{n=1}^{N}\left(X^{B}(n)-A\right)\right|_{f_{n}} \text { as shown below } \\
& =X-\left(X^{B}-A\right) \text { by the definition of } X^{B} \\
& =X^{S} \text { by the definition of } X^{S} .
\end{aligned}
$$

To see the fourth equality, observe that

$$
\bigcup_{n=1}^{N}\left(X^{B}(n)-\left.A\right|_{f_{n}}\right)=X^{B}-A=R_{B}\left(X^{B}(N)\right) .
$$

Suppose that there exists a nonempty set of contracts

$$
Y \subseteq \bigcup_{n=1}^{N}\left(X^{B}(n)-\left.A\right|_{f_{n}}\right)-\bigcup_{n=1}^{N} R_{B}^{f_{n}}\left(X^{B}(n) \mid X^{S}(n)\right)
$$

We also know that no contract $Y$ is rejected by a seller (assuming sellers have access to $X^{B}$ as buyers) as these are contracts $X^{B}$. Hence, $Y$ is a blocking set and hence $A$ is not stable, a contradiction. Finally, $\left.A\right|_{f_{n}} \cap R_{B}^{f}\left(X^{B}(n) \mid X^{S}(n)\right)=\varnothing$ as $A$ is individually rational.

Finally, we need to show that $X^{B} \cap X^{S}=A$. First, since $X^{B}(0) \cap X^{S}(0)=A$ and $X^{B}(n-1) \subseteq X^{B}(n)$ and $X^{S}(n-1) \subseteq X^{S}(n), A \subseteq X^{B}(0) \cap X^{S}(0)$. Suppose that $z \in X^{S}-A$. Then $z \notin X^{B}$, as it could only be added in the $z_{B}$-th step and since $z \in X^{S}, a \in X^{S}\left(f_{z_{B}}-1\right)$. 
For any stable allocation $\left(X^{B}, X^{S}\right)$, we have that

$$
\begin{aligned}
X^{B} \cap X^{S} & =X^{B} \cap\left(X-R_{B}\left(X^{B} \mid X^{S}\right)\right) \\
& =X^{B} \cap\left(X-\left(X^{B}-C_{B}\left(X^{B} \mid X^{S}\right)\right)\right) \\
& =X^{B}-\left(X^{B}-C_{B}\left(X^{B} \mid X^{S}\right)\right) \\
& =C_{B}\left(X^{B} \mid X^{S}\right) .
\end{aligned}
$$

For each firm $f$ who is only a buyer, $C_{B}^{f}\left(X^{B} \mid X^{S}\right)=C^{f}\left(X^{B}\right)$, the firm $f$ has a strictly larger choice set under $\left(\hat{X}^{B}, \hat{X}^{S}\right)$ than under any other stable allocation, and hence (weakly) prefers $\left(\hat{X}^{B}, \hat{X}^{S}\right)$. The proof that $\left(\check{X}^{B}, \check{X}^{S}\right)$ is the seller-optimal stable allocation is symmetric.

\section{Proof of Theorem 5}

Let $y$ be the contract between $g$ and some member of the cycle. Without loss of generality, we suppose that $f_{n} \equiv x_{S}^{n}, y_{S}=f_{1}$, and $y_{B}=g$. Then for $n=2, \ldots, N$, let the preferences of firm $f_{n}$ be

$$
P^{f_{n}}:\left\{x^{n-1}, x^{n}\right\} \succ \varnothing
$$

which are fully substitutable. Let

$$
\begin{aligned}
P^{g} & :\{y\} \succ \varnothing \\
P^{f_{1}} & :\left\{x^{N}, y\right\} \succ\left\{x^{N}, x^{1}\right\} \succ \varnothing ;
\end{aligned}
$$

these preferences are fully substitutable. Let all other firms desire no contracts. Any set $Y \nsubseteq L \cup\{y\}$ is not stable, as it is not individually rational. Any $Z \subsetneq L \cup\{y\}$ is not stable, as it not individually rational unless $Z=\varnothing$, in which case $L$ is a blocking set, or $Z=L$, in which case $\{y\}$ is a blocking set. Finally, $L \cup\{y\}$ is not stable, as it is not individually rational for $f_{1}$.

\section{Proof of Theorem 6}

If the preferences of a firm $f$ are not same-side substitutable, then there exist contracts $x, y,\left.z \in X\right|_{f}$ and sets of contracts $Y, Z \subseteq X$ such that $Y_{B}=\{f\}$ and $Z_{S}=\{f\}$ such 
that

$$
\begin{aligned}
& y \notin C_{B}^{f}(Y \mid Z) \text { but } y \in C_{B}^{f}(\{x\} \cup Y \mid Z) \text { or } \\
& z \notin C_{S}^{f}(Z \mid Y) \text { but } z \in C_{S}^{f}(\{x\} \cup Z \mid Y)
\end{aligned}
$$

Assume the former; the latter case is symmetric. There are two cases.

Case 1: $x_{S} \neq y_{S}$. By assumption, there must exist a firm $g$ that is downstream of $f$ and hence downstream of both $x_{S}$ and $y_{S}$. Furthermore, by exhaustivity, there must exist contracts $\hat{x}$ and $\hat{y}$ with $x_{S}=\hat{x}_{S}, y_{S}=\hat{y}_{S}$ and $\hat{x}_{B}=\hat{y}_{B}=g$. Let $y_{S}$ have preferences such that

$$
C^{y_{S}}(W)=\left\{\begin{array}{ll}
\left.(W \cap(Y \cup Z))\right|_{y_{S}} & \{y, \hat{y}\} \subseteq W \\
\left.(W \cap(Y \cup Z \cup\{\hat{y}\}))\right|_{y_{S}} & \text { otherwise }
\end{array} .\right.
$$

That is, $y_{S}$ is willing to accept any and all of the contracts the firm is associated with in $Y \cup Z-\{y\}$, and $y_{S}$ wants one of $y$ and $\hat{y}$, preferring $y$, and rejects all other contracts. Let $x_{S}$ have preferences such that

$$
C^{x_{S}}(W)=\left\{\begin{array}{ll}
\left.(W \cap(Y \cup Z \cup\{\hat{x}\}))\right|_{x_{S}} & \{x, \hat{x}\} \subseteq W \\
\left.(W \cap(\{x\} \cup Y \cup Z \cup\{\hat{x}\}))\right|_{x_{S}} & \text { otherwise }
\end{array} .\right.
$$

Let $g$ have preferences such that

$$
C^{g}(W)=\left\{\begin{array}{ll}
\left.(W \cap(Y \cup Z \cup\{\hat{y}\}))\right|_{g} & \{\hat{y}, \hat{x}\} \subseteq W \\
\left.(W \cap(Y \cup Z \cup\{\hat{y}, \hat{x}\}))\right|_{g} & \text { otherwise }
\end{array} .\right.
$$

Let every firm $\tilde{f} \in F-\left\{x_{S}, y_{S}, f, g\right\}$ have preferences such that

$$
C^{\tilde{f}}(W)=\left.(W \cap(Y \cup Z))\right|_{\tilde{f}}
$$

Consider any allocation $A$; we will show $A$ can not be stable.

1) Suppose $\left.A\right|_{f} \prec_{f} C^{f}(Y \cup Z)$. If $A$ is individually rational, then all other firms want their contracts in $C^{f}(Y \cup Z)$ irrespective of their other contracts, so $C^{f}(Y \cup Z)$ blocks $A$.

2) Suppose $\left.A\right|_{f}=C^{f}(Y \cup Z)$. Then $\hat{y} \in A$, as otherwise $\{\hat{y}\}$ blocks $A$. But then $C^{f}(\{x\} \cup Y \cup Z)$ blocks $A$.

3) Suppose $\left.C^{f}(\{x\} \cup Y \cup Z) \succ_{f} A\right|_{f} \succ_{f} C^{f}(Y \cup Z)$. In this case, if $A$ is individually rational, then $A \subseteq\{x, \hat{x}, \hat{y}\} \cup Y \cup Z$; then $x \in A$ as otherwise we could not have $\left.A\right|_{f} \succ_{f} C^{f}(Y \cup Z)$. But then, $C^{f}(\{x\} \cup Y \cup Z)$ blocks A. 
4) Suppose $C^{f}(\{x\} \cup Y \cup Z)=\left.A\right|_{f}$. Then if $\hat{y} \in A$, the allocation $A$ is not individually rational for $y_{S}$, and if $\hat{x} \in A$, the allocation $A$ is not individually rational for $x_{S}$; but this implies that $\{\hat{x}\}$ blocks $A$.

Case 2: $x_{S}=y_{S} \equiv d$. By assumption, there are two firms, $g$ and $h$, downstream of $f$, and one firm, $e$, upstream of $f$, and hence upstream of $g$ and $h$. Now consider the contracts $v, v^{\prime}, w$ and $w^{\prime}$ such that $v_{S}=w_{S}=d, v_{S}^{\prime}=w_{S}^{\prime}=e, v_{B}=v_{B}^{\prime}=g$ and $w_{B}=w_{B}^{\prime}=h$ (which exist as $X$ is exhaustive). Let $d$ have preferences such that

$$
C^{d}(W)=\left.(W \cap((Y-\{y\}) \cup Z))\right|_{d} \cup \tilde{C}^{d}(W \cap\{x, y, w, v\})
$$

where $\tilde{C}^{d}(\tilde{W})$ is the responsive choice function over $\{x, y, w, v\}$ with quota 2 and underlying preference order $w \succ y \succ x \succ v$. Let $e, g, h$ have preferences such that

$$
\begin{aligned}
& C^{e}(W)= \begin{cases}\left(\left.W \cap\left(Y \cup Z \cup\left\{v^{\prime}\right\}\right)\right|_{e}\right. & \left\{v^{\prime}, w^{\prime}\right\} \subseteq W \\
\left.\left(W \cap\left(Y \cup Z \cup\left\{v^{\prime}, w^{\prime}\right\}\right)\right)\right|_{e} & \text { otherwise }\end{cases} \\
& C^{g}(W)= \begin{cases}\left.(W \cap(Y \cup Z \cup\{v\}))\right|_{g} & \left\{v, v^{\prime}\right\} \subseteq W \\
\left.\left(W \cap\left(Y \cup Z \cup\left\{v, v^{\prime}\right\}\right)\right)\right|_{g} & \text { otherwise }\end{cases} \\
& C^{h}(W)= \begin{cases}\left.\left(W \cap\left(Y \cup Z \cup\left\{w^{\prime}\right\}\right)\right)\right|_{h} & \left\{w, w^{\prime}\right\} \subseteq W \\
\left.\left(W \cap\left(Y \cup Z \cup\left\{w, w^{\prime}\right\}\right)\right)\right|_{h} & \text { otherwise }\end{cases}
\end{aligned}
$$

Finally, let every firm $\tilde{f} \in F-\{d, e, f, g, h\}$ have preferences such that

$$
C^{\tilde{f}}(W)=\left.(W \cap(Y \cup Z))\right|_{\tilde{f}} .
$$

Consider any allocation $A$; we will show $A$ can not be stable.

1) Suppose $\left.A\right|_{f} \prec_{f} C^{f}(Y \cup Z)$. Then $C^{f}(Y \cup Z)$ blocks $A$, as all the firms want their contracts in $C^{f}(Y \cup Z)$, irrespective of other contracts.

2) Suppose $\left.A\right|_{f}=C^{f}(Y \cup Z)$. Since $d$ does not obtain $x$ or $y$, he desires both $v$ and $w$. If $A$ is stable then, $v \in A$. Furthermore, since $e$ does not obtain $v^{\prime}$, for $A$ to be stable, then $w^{\prime} \in A$. Hence, if $A$ is stable, $\left\{w^{\prime}, v\right\} \subseteq A$ and $w \notin A$. In that case, $C^{f}(\{x\} \cup Y \cup Z)$ blocks $A$.

3) Suppose $\left.C^{f}(Y \cup Z) \prec_{f} A\right|_{f} \prec C^{f}(\{x\} \cup Y \cup Z)$. Then $x \in A$, so $C^{f}(\{x\} \cup Y \cup Z)-\{x\}$ blocks $A$, as $d$ will always choose $y$ and the other firms in $Y$ and $Z$ will always accept offers of any and all contracts in $Y$.

4) Suppose $C^{f}(\{x\} \cup Y \cup Z)=\left.A\right|_{f}$. If $v^{\prime} \notin A$, then $\left\{v^{\prime}\right\}$ blocks $A$. (Note that if $v \in A$, then $\{x, y, v\} \subseteq A$, and so $A$ is not individually rational for $d$.) But $v^{\prime} \in A$ implies that $w^{\prime} \notin A$. Hence $\{w\}$ blocks $A$. (Note that $w \notin A$, as then $\{w, x, y\} \subseteq A$, and so $A$ is not individually rational for $d$.) 
If the preferences of a firm $f$ are not cross-side complementary, then there exists contracts $y, z \in X$ and sets of contracts $Y, Z \subseteq X-\{y, z\}$ such that $Y_{B}=\{f\}$ and $Z_{S}=\{f\}$ such that

$$
\begin{aligned}
& y \in C_{B}^{f}(\{y\} \cup Y \mid Z) \text { but } y \notin C_{B}^{f}(\{y\} \cup Y \mid\{z\} \cup Z) \text { or } \\
& z \in C_{S}^{f}(\{z\} \cup Z \mid Y) \text { but } z \notin C_{S}^{f}(\{z\} \cup Z \mid\{y\} \cup Y) .
\end{aligned}
$$

Assume the latter; the former case is symmetric.

By exhaustivity, there must exist a contract $w \in X$ such that $w_{S}=y_{S}$ and $w_{B}=z_{B}$. Let $y_{S}$ have preferences such that

$$
C^{y_{S}}(W)=\left\{\begin{array}{ll}
\left.(W \cap(Y \cup Z \cup\{w\}))\right|_{y_{S}} & \{w, y\} \subseteq W \\
\left.(W \cap(\{y\} \cup Y \cup Z \cup\{w\}))\right|_{y_{S}} & \text { otherwise }
\end{array} .\right.
$$

Let $z_{B}$ have preferences such that

$$
C^{z_{B}}(W)=\left\{\begin{array}{ll}
\left.(W \cap(Y \cup Z \cup\{z\}))\right|_{z_{B}} & \{z, w\} \subseteq W \\
\left.(W \cap(Y \cup Z \cup\{z, w\}))\right|_{z_{B}} & \text { otherwise }
\end{array} .\right.
$$

Finally, let every firm $\tilde{f} \in F-\left\{f, y_{S}, z_{B}\right\}$ have preferences such that

$$
C^{\tilde{f}}(W)=\left.(W \cap(Y \cup Z))\right|_{\tilde{f}}
$$

Consider any allocation $A$; we will show $A$ can not be stable.

1) Suppose $\left.A\right|_{f} \prec_{f} C^{f}(Y \cup\{z\} \cup Z)$. If $A$ is individually rational, all other firms want their contracts in $C^{f}(Y \cup\{z\} \cup Z)$ irrespective of their other contracts, so $C^{f}(Y \cup\{z\} \cup Z)$ blocks $A$.

2) Suppose $\left.A\right|_{f}=C^{f}(Y \cup\{z\} \cup Z)$. Then, if $A$ is individually rational, $w \notin A$, as $z \in C^{f}(Y \cup\{z\} \cup Z)$. But then $C^{f}(\{y\} \cup Y \cup\{z\} \cup Z)$ blocks $A$.

3) Suppose $\left.C^{f}(\{y\} \cup Y \cup\{z\} \cup Z) \succ_{f} A\right|_{f} \succ_{f} C^{f}(Y \cup\{z\} \cup Z)$. In this case, $y \in A$ as otherwise $\left.A\right|_{f}$ is available to $f$ from $Y \cup\{z\} \cup Z$, so we could not have $\left.A\right|_{f} \succ_{f} C^{f}(Y \cup\{z\} \cup Z)$. But then, $C^{f}(\{y\} \cup Y \cup\{z\} \cup Z)$ blocks $A$.

4) Suppose $C^{f}(\{y\} \cup Y \cup\{z\} \cup Z)=\left.A\right|_{f}$. Then if $w \in A$, $A$ is not individually rational for $z_{B}$; if $w \notin A,\{w\}$ blocks $A$.

\section{Proof of Theorem 7}

Consider an allocation $A$ that is not stable. If $A$ is not individually rational, then $A$ is not chain stable. Hence, suppose there is a blocking set $Z$ for $A$. Since $X$ is acyclic, 
there is an ordering of firms in $Z_{F}$ such that $x_{B} \triangleright x_{S}$ for all $x \in X$. Consider a firm $f \in Z_{F}$ such that $f \triangleright g$ for all $g \in Z_{F}$, and consider one contract $y^{1} \in Z$ such that $y_{B}^{1}=f$. Now consider $y_{S}^{1}$. By same-side substitutability, $y^{1} \in C_{B}^{y_{B}^{1}}\left(\left\{y^{1}\right\} \cup A \mid A\right)$ and $y^{1} \in C_{S}^{y_{S}^{1}}\left(\left\{y^{1}\right\} \cup A \mid A \cup Z\right)$. If $y^{1} \in C_{S}^{y_{S}^{1}}\left(\left\{y^{1}\right\} \cup A \mid A\right)$, then the chain $\left\{y^{1}\right\}$ is a blocking set and we are done. If not, then there exists a contract $y^{2} \in Z$ such that $y^{1} \in C_{S}^{y_{S}^{1}}\left(\left\{y^{1}\right\} \cup A \mid A \cup\left\{y^{2}\right\}\right)$ and $y^{2} \in C_{B}^{y_{S}^{1}}\left(\left\{y^{2}\right\} \cup A \mid A \cup\left\{y^{1}\right\}\right)$, by same-side substitutability. (If no such $y^{2}$ exists, then preferences of $y_{S}^{1}$ are not fully substitutable, as there exists $y^{2} \notin C_{B}^{y_{S}^{1}}\left(\left\{y^{2}\right\} \cup A \mid A \cup\left\{y^{1}\right\}\right)$, but $y^{2} \in C_{B}^{y_{S}^{1}}\left(Z \cup A \mid A \cup\left\{y^{1}\right\}\right)$.) By an argument analogous to that we provided for $y^{1}$, we see that either the chain $\left\{y^{1}, y^{2}\right\}$ is a blocking set, or there exists $y^{3} \in Z$ such that $y^{2}, y^{3} \in C^{y_{S}^{2}}\left(\left\{y^{1}, y^{2}, y^{3}\right\} \cup A\right)$. Iterating this argment, we find a chain $\left\{y^{1}, y^{2}, \ldots, y^{n}\right\}$ blocking $A$, as $Z$ is a finite set.

\section{Proof of Theorem 8}

Consider any stable allocation $A$ associated with the fixed point $\left(X^{S}, X^{B}\right)$ and the seller-optimal stable allocation $\hat{A}$ associated with the fixed point $\left(\hat{X}^{S}, \hat{X}^{B}\right)$. Consider an arbitrary firm $f$. We have that

$$
\begin{aligned}
\left|C_{S}^{f}\left(\hat{X}^{S} \mid \hat{X}^{B}\right)\right|-\left|C_{B}^{f}\left(\hat{X}^{B} \mid \hat{X}^{S}\right)\right| & \geq\left|C_{S}^{f}\left(X^{S} \mid \hat{X}^{B}\right)\right|-\left|C_{B}^{f}\left(\hat{X}^{B} \mid X^{S}\right)\right| \\
& \geq\left|C_{S}^{f}\left(X^{S} \mid X^{B}\right)\right|-\left|C_{B}^{f}\left(X^{B} \mid X^{S}\right)\right|,
\end{aligned}
$$

where the first inequality follows by the Law of Aggregate Supply, as $\hat{X}^{S} \supseteq X^{S}$, and the second follows by the law of aggregate demand, as $\hat{X}^{B} \subseteq X^{B}$. Hence, the difference between the number of contracts $f$ sells and the number $f$ buys is weakly greater under $\left(\hat{X}^{S}, \hat{X}^{B}\right)$ than $\left(X^{S}, X^{B}\right)$. However, the sum across all firms of the difference between the number of contracts bought and the number of contracts sold equals 0 . It follows that, for each firm,

$$
\left|C_{S}^{f}\left(\hat{X}^{S} \mid \hat{X}^{B}\right)\right|-\left|C_{B}^{f}\left(\hat{X}^{B} \mid \hat{X}^{S}\right)\right|=\left|C_{S}^{f}\left(X^{S} \mid X^{B}\right)\right|-\left|C_{B}^{f}\left(X^{B} \mid X^{S}\right)\right| .
$$

\section{Proof of Theorem 9}

Since the contractual set $X$ is acyclic, there is an ordering $\triangleleft$ on firms such that $x_{S} \triangleleft$ $x_{B}$ for all $x \in X$. By symmetry, we need only consider the following three possible cases.

1) There exist contracts $x, y, z \in X$ and sets $Y, Z \subseteq X$ such that $Y_{B}=\{f\}$ and $Z_{S}=\{f\}$ such that

$$
x, y \in C_{S}^{f}(Z \mid Y) \text { and } x, y \notin C_{S}^{f}(\{z\} \cup Z \mid Y) .
$$


Let every firm $\tilde{f} \in F-\left\{x_{B}, y_{B}, z_{B}, f\right\}$ have preferences such that

$$
C^{\tilde{f}}(W)=\left.(W \cap(Y \cup Z))\right|_{\tilde{f}} .
$$

There are two subcases to consider:

a) We have either $f \triangleleft x_{B} \triangleleft z_{B}$ or $f \triangleleft y_{B} \triangleleft z_{B}$; assume the former (the latter is symmetric). By exhaustivity of $X$, there exist contracts $\hat{y}, \hat{z} \in X$ such that $\hat{y}_{B}=y_{B}, \hat{z}_{B}=z_{B}$, and $\hat{y}_{S}=\hat{z}_{S}=x_{B}$. Let $x_{B}, y_{B}, z_{B}$ have preferences such that

$$
\begin{aligned}
& C^{x_{B}}(W)= \begin{cases}\left.(W \cap(\{x\} \cup Y \cup Z \cup\{\hat{y}\}))\right|_{x_{B}} & \{\hat{y}, \hat{z}\} \subseteq W \\
\left.(W \cap(\{x\} \cup Y \cup Z \cup\{\hat{y}, \hat{z}\}))\right|_{x_{B}} & \text { otherwise }\end{cases} \\
& C^{y_{B}}(W)= \begin{cases}\left.(W \cap(Y \cup Z \cup\{y\}))\right|_{y_{B}} & \{y, \hat{y}\} \subseteq W \\
\left.(W \cap(Y \cup Z \cup\{y, \hat{y}\}))\right|_{y_{B}} & \text { otherwise }\end{cases} \\
& C^{z_{B}}(W)=\left\{\begin{array}{ll}
\left.(W \cap(Y \cup Z \cup\{\hat{z}\}))\right|_{z_{B}} & \{\hat{z}, z\} \subseteq W \\
\left.(W \cap(Y \cup Z \cup\{\hat{z}, z\}))\right|_{z_{B}} & \text { otherwise }
\end{array} .\right.
\end{aligned}
$$

There are at least two stable allocations: $C^{f}(Z \cup Y) \cup\{\hat{z}\}$ and $C^{f}(\{z\} \cup$ $Z \cup Y) \cup\{\hat{y}\}$. By the cross-side compelementarity of the preferences of $f,\left|C_{B}^{f}(Y \mid Z)\right| \geq\left|C_{B}^{f}(Y \mid\{z\} \cup Z)\right|$ and the same-side subsitutability of the preferences of $f,\left|C_{S}^{f}(Z \mid Y)\right|<\left|C_{S}^{f}(\{z\} \cup Z \mid Y)\right|$, and hence the conclusion of Theorem 8 fails for $f$.

b) We have $f \triangleleft z_{B} \triangleleft x_{B}, y_{B}$. By exhaustivity of $X$, there exists a contract $\hat{x} \in X$ such that $\hat{x}_{S}=z_{B}$ and $\hat{x}_{B}=x_{B}$. Let $x_{B}, y_{B}, z_{B}$ have preferences such that

$$
\begin{aligned}
& C^{x_{B}}(W)= \begin{cases}\left.(W \cap(Y \cup Z \cup\{x\}))\right|_{x_{B}} & \{x, \hat{x}\} \subseteq W \\
\left.(W \cap(Y \cup Z \cup\{x, \hat{x}\}))\right|_{x_{B}} & \text { otherwise }\end{cases} \\
& C^{y_{B}(W)=\left.(W \cap(Y \cup Z \cup\{y\}))\right|_{y_{B}}} \\
& C^{z_{B}}(W)=\left\{\begin{array}{ll}
\left.(W \cap(Y \cup Z \cup\{\hat{x}, z\}))\right|_{z_{B}} & \{\hat{x}, z\} \subseteq W \\
\left.(W \cap(Y \cup Z))\right|_{z_{B}} & \text { otherwise }
\end{array} .\right.
\end{aligned}
$$

There are at least two stable allocations: $C^{f}(Z \cup Y)$ and $C^{f}(\{z\} \cup Z \cup Y) \cup$ $\{\hat{x}\}$, and hence (again by the full substitutability of the preferences of $f$ as above) the conclusion of Theorem 8 fails for $f$.

2) There exist contracts $x, y, z \in X$ and sets $Y, Z \subseteq X$ such that $Y_{B}=\{f\}$ and 
$Z_{S}=\{f\}$ such that

$$
x \in C_{S}^{f}(Z \mid Y), y \notin C_{B}^{f}(Y \mid Z) \text { and } x \notin C_{S}^{f}(\{z\} \cup Z \mid Y), y \in C_{B}^{f}(Y \mid\{z\} \cup Z)
$$

By exhaustivity of $X$, there exists a contract $\hat{y}$ such that $\hat{y}_{S}=y_{S}$ and $\hat{y}_{B}=z_{B}$. Let $x_{B}, y_{B}, z_{B}$ have preferences such that

$$
\begin{aligned}
& C^{x_{B}}(W)=\left.(W \cap(\{x\} \cup Y \cup Z))\right|_{x_{B}} \\
& C^{y_{B}}(W)= \begin{cases}\left.(W \cap(Y \cup Z \cup\{y\}))\right|_{y_{B}} & \{y, \hat{y}\} \subseteq W \\
\left.(W \cap(Y \cup Z \cup\{y, \hat{y}\}))\right|_{y_{B}} & \text { otherwise }\end{cases} \\
& C^{z_{B}}(W)= \begin{cases}\left.(W \cap(Y \cup Z \cup\{\hat{y}\}))\right|_{z_{B}} & \{\hat{y}, z\} \subseteq W \\
\left.(W \cap(Y \cup Z \cup\{\hat{y}, z\}))\right|_{z_{B}} & \text { otherwise }\end{cases}
\end{aligned}
$$

Finally, let every firm $\tilde{f} \in F-\left\{x_{B}, y_{B}, z_{B}, f\right\}$ have preferences such that

$$
C^{\tilde{f}}(W)=\left.(W \cap(Y \cup Z))\right|_{\tilde{f}} .
$$

There are at least two stable allocations: $C^{f}(Z \cup Y) \cup\{\hat{y}\}$ and $C^{f}(\{z\} \cup Z \cup Y)$, and hence (again by the full substitutability of the preferences of $f$ as above) the conclusion of Theorem 8 fails for $f$.

3) There exist contracts $x, y, z \in X$ and sets $Y, Z \subseteq X$ such that $Y_{B}=\{f\}$ and $Z_{S}=\{f\}$ such that

$$
x, y \notin C_{B}^{f}(Y \mid Z) \text { and } x, y \in C_{B}^{f}(Y \mid\{z\} \cup Z) .
$$

By exhaustivity of $X$, there exists a contract $\hat{y}$ such that $\hat{y}_{S}=y_{S}$ and $\hat{y}_{B}=z_{B}$. The same preferences constructed in the previous case again render at least two allocations stable: $C^{f}(Z \cup Y) \cup\{\hat{y}\}$ and $C^{f}(\{z\} \cup Z \cup Y)$, and hence the conclusion of Theorem 8 fails for $f$. 